\title{
Ferrier sulfamidoglycosylation of glycals catalyzed by nitrosonium tetrafluoroborate: Towards new carbonic anhydrase glycoinhibitors
}

\author{
Joanna Ombouma ${ }^{a}$, Daniela Vullo ${ }^{b}$, Claudiu T. Supuran ${ }^{c}$, Jean-Yves Winum ${ }^{a, *}$ \\ ${ }^{a}$ Institut des Biomolécules Max Mousseron (IBMM), UMR 5247 CNRS-UM1-UM2, Bâtiment de Recherche Max Mousseron, Ecole Nationale Supérieure de Chimie de Montpellier, \\ 8 rue de l'Ecole Normale, 34296 Montpellier Cedex, France \\ ${ }^{\mathrm{b}}$ Laboratorio di Chimica Bioinorganica, Polo Scientifico, Università degli Studi di Firenze, Sesto Fiorentino, Florence, Italy \\ ${ }^{\mathrm{c}}$ Neurofarba Department, Section of Pharmaceutical and Nutraceutical Sciences, Università degli Studi di Firenze, Sesto Fiorentino, Florence, Italy
}

\section{A R T I C L E I N F O}

Article history:

Received 25 August 2014

Revised 17 September 2014

Accepted 25 September 2014

Available online 2 October 2014

\section{Keywords:}

Ferrier sulfamidoglycosylation

Glycals

Nitrosonium tetrafluoroborate

Glycoinhibitor

Carbonic anhydrase

\begin{abstract}
A B S T R A C T
Ferrier sulfamidoglycosylation of glycals catalyzed by nitrosonium tetrafluoroborate allowed the preparation of hydroxysulfamide glycosides in good yields with a good $\alpha$ stereoselectivity. A variety of monosaccharide derivatives was synthesized using this new methodology leading to selective and powerful glycoinhibitors of the tumor associated carbonic anhydrases (CA, EC 4.2.1.1) isoforms CA IX and CA XII.
\end{abstract}

(c) 2014 Elsevier Ltd. All rights reserved.

\section{Introduction}

The development of carbonic anhydrases (CA, EC 4.2.1.1) glycoinhibitors is currently a dynamic field which constitutes one of the most successful approaches to find new active and selective inhibitors with potent biomedical applications. ${ }^{1}$ Carbohydrate based CA inhibitors were already found to be effective as antiglaucoma or anti-epileptic agents. ${ }^{1}$ Some recent work from our group also demonstrated the validity of this approach in the field of cancer as we were able to demonstrate that carbonic anhydrase inhibitors belonging to a glycosylcoumarin series were strong, selective CA IX inhibitors and were able to reduce the growth of primary tumors and metastases in a human and mouse model of orthotopic, CA IX-positive breast cancer ${ }^{2}$ and also to deplete cancer stem cells within these tumors. ${ }^{3}$ In our ongoing researches in the development of new and original glycoinhibitors incorporating the hydroxysulfamide scaffold as zinc binding function, ${ }^{4}$ we were interested to find a synthetic methodology allowing the access to 2,3-unsaturated glycosides $\mathbf{5}$ starting from a peracetylated glycals platform (Scheme 1).

Based on previous works reported by Colinas and Bravo ${ }^{5}$ describing the Ferrier sulfonamidoglycosylation of glycals, we sought to extend this methodology to sulfamides using the

\footnotetext{
* Corresponding author.

E-mail address: jean-yves.winum@univ-montp2.fr (J.-Y. Winum).
}

non-metallic catalyst nitrosyl tetrafluoroborate. Indeed, $\mathrm{NOBF}_{4}$ was recently demonstrated by Misra and Coll. ${ }^{6}$ to be efficient for the stereoselective glycosylation reaction as well as for the preparation of 2,3-unsaturated glycosides and 2-deoxyglycosides. In this paper, we report our findings on this reaction and also the inhibitory activity of the obtained $N$-glycosyl- $N$-hydroxysulfamides against four relevant CA isoforms.

\section{Results and discussion}

We first focused our attention to the conditions for the Ferrier sulfamidoglycosylation of the tri-O-acetyl-D-glucal with $\mathrm{N}-(\mathrm{O}$ tert-butyldiphenylsilyl)hydroxysulfamide $\mathbf{1}$, prepared initially from the commercial hydroxylamine hydrochloride, following a
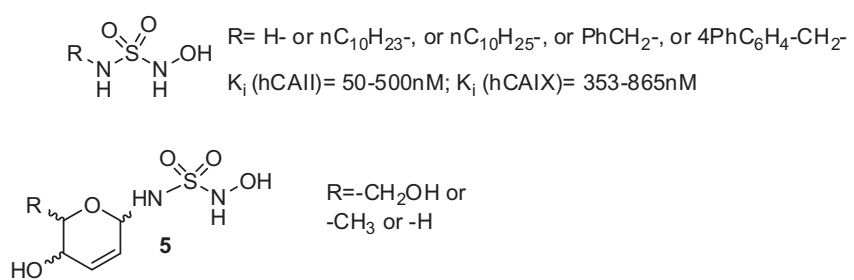

Scheme 1. Structure of hydroxysulfamide CA inhibitors previously described ${ }^{4}$ and general structure of the targeted compound $\mathbf{5}$ 


$$
\begin{aligned}
& \text { 1) TBDPSCI, } \mathrm{NEt}_{3}, 0^{\circ} \mathrm{C}, \mathrm{CH}_{2} \mathrm{Cl}_{2} \\
& \mathrm{NH}_{2}-\mathrm{OH}, \mathrm{HCl} \\
& \text { 2) } \mathrm{CSI}, \mathrm{tBuOH}, 0^{\circ} \mathrm{C}, \mathrm{CH}_{2} \mathrm{Cl}_{2} \\
& \text { 3) } 10 \% \text { TFA, } \mathrm{CH}_{2} \mathrm{Cl}_{2}
\end{aligned}
$$

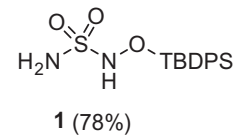

Scheme 2. Synthesis of $N$-(O-tert-butyldiphenylsilyl)hydroxysulfamide $\mathbf{1}$.

three steps synthesis as depicted in Scheme 2. The hydroxylamine was first $\mathrm{O}$-protected with the tert-butyldiphenylsilyl protecting group, and then sulfamoylated with tert-butoxysulfamoyl chloride, prepared ab initio by reacting chlorosulfonyl isocyanate with tertbutanol. Final Boc deprotection with 10\% TFA solution in methylene chloride allowed formation of compound $\mathbf{1}$ in an overall yield of $78 \%{ }^{7}$

First attempts of sulfamidoglycosylation of glycal $\mathbf{2}$ was realized using the classical Lewis acid $\mathrm{BF}_{3} \cdot \mathrm{Et}_{2} \mathrm{O}$, widely used as catalyst in Ferrier rearrangement of glycals and described in 2007 by Colinas research group as a good catalyst of the Ferrier sulfonamidoglycosylation of D-glycals. ${ }^{5}$ Unfortunately, low reaction yields (around $20 \%$ ), obtained with this catalyst, led us to use $\mathrm{NOBF}_{4}$, recently reported as mild, efficient and inexpensive catalyst for the Ferrier rearrangement reaction. ${ }^{6}$

In order to improve in term of efficiency, selectivity, time and yield of reaction we investigated the reaction in the presence of a variable amount of $\mathrm{NOBF}_{4}$, at different temperatures. We found that the reaction proceeded effectively at $50{ }^{\circ} \mathrm{C}$ using 1.1 equiv of 1 and 0.05 equiv of $\mathrm{NOBF}_{4}$ in methylene chloride. Other organic solvents were tested (e.g., acetonitrile, chloroform), but no improvements in terms of yields and reaction time were observed.

The reaction was also subjected to microwave irradiation instead of thermal activation. In this case, an overall $50 \%$ reduction of the reaction time was possible.

These optimized conditions were applied on a variety of glycals such as D-galactal, D-arabinal, L-arabinal and L-rhamnal. (Scheme 3) and the corresponding Ferrier sulfamidoglycosylation products $\mathbf{3}$ were obtained in moderate to fairly good yields. In all cases, a mixture of non-separable $\alpha$ - and $\beta$-anomers was obtained, with the $\alpha$ anomer being predominant in all cases (Table 1), as it was observed also in the case of sulfonamidoglycosylation reactions reported by Colinas and Bravo. ${ }^{5}$

Removal of the silyl protecting groups from compound $\mathbf{3}$ was initially performed by standard methods using either tetra- $n$-butyl ammonium fluoride $(\mathrm{TBAF})^{8}$, or Selectfluor ${ }^{\mathrm{TM}}{ }^{9}$ and led to low yields ( $\sim 20 \%$ ) of the corresponding compound 4. Ultimately deprotection

\begin{tabular}{|c|c|c|}
\hline Glycals 1 & $\begin{array}{l}\text { Step } 1^{\text {a }} \\
\text { Yield } \% / \alpha \mid \beta \text { ratio/reaction time }\end{array}$ & $\begin{array}{l}\text { Steps } 2+3^{\mathrm{b}} \\
\text { Yield } \% / \alpha / \beta \text { ratio }\end{array}$ \\
\hline $2 a$ & $\begin{array}{l}\Delta: 68 / 60: 40 / 45 \min \\
\text { MW: } 70 / 60: 40 / 20 \min \end{array}$ & $85 / 77: 23$ \\
\hline 2b & $\begin{array}{l}\Delta: 70 / 75: 25 / 45 \min \\
\text { MW: 70/75:25/20 min }\end{array}$ & $85 / 77: 23$ \\
\hline 2c & $\begin{array}{l}\Delta: 88 / 60: 40 / 45 \min \\
\text { MW: } 90 / 60: 40 / 20 \min \end{array}$ & $85 / 77: 23$ \\
\hline $2 d$ & $\begin{array}{l}\Delta: 86 / 60: 40 / 45 \min \\
\text { MW: } 90 / 60: 40 / 20 \min \end{array}$ & $85 / 77: 23$ \\
\hline $2 e$ & $\begin{array}{l}\Delta: 88 / 84: 16 / 45 \min \\
\text { MW: } 90 / 84: 16 / 20 \min \end{array}$ & $85 / 77: 23$ \\
\hline
\end{tabular}
of the silyl ether was carried out using HF-pyridine complex and
Table 1

Optimized results for the Ferrier sulfamidoglycosylation and deprotection steps

a Reaction conditions: glycal (1) 1 equiv $\mathrm{N}$-(O-tert-butyldiphenylsilyl)hydroxysulfamide 1.1 equiv, $\mathrm{NOBF}_{4} 0.05$ equiv, $\mathrm{CH}_{2} \mathrm{Cl}_{2}$.

b HF-pyridine $70 \%, \mathrm{CH}_{3} \mathrm{CN}$, RT, then $\mathrm{NH}_{3} / \mathrm{MeOH}$.

compound 4 were obtained in $85 \%$ yields. ${ }^{10}$ Final cleavage of the acetyl group was done by using a methanolic solution of ammonia, to afford quantitatively compound $\mathbf{5}$.

The structure of compounds $\mathbf{1}, \mathbf{3}, \mathbf{4}$ and $\mathbf{5}$, as well as the ratio of $\alpha / \beta$ anomers were unambiguously confirmed using ${ }^{1} \mathrm{H},{ }^{13} \mathrm{C}, 2 \mathrm{D}$ COSY pulseprog COSYGPQF (with gradient quadrature mode; time domain size, $\mathrm{TD}=2 \mathrm{k}$; relaxation delay, $\mathrm{d} 1=1.5 \mathrm{~s}$ and scan number $=1$ ), HMQC experiments.

Stereochemical assignment of the major diastereoisomer was verified by NOESY pulseprog NOESYGPPHPP experiments (time domain size, $\mathrm{TD}=2 \mathrm{k}$; the mixing time $\mathrm{d} 8=0.7 \mathrm{~s}$; the relaxation delay $\mathrm{d} 1=1.5 \mathrm{~s}$ and scans number $=16$ ), observing a NOE interaction between $\mathrm{H}_{1}$ and $\mathrm{H}_{5}$ for the $\beta$-anomers (absent for the $\alpha$ anomers).

At this point, we can provide two plausible mechanisms for the sulfamidoglycosylation reaction of glycals, inspired by previous work reported by Ansari et al. ${ }^{11}$ and Toshima et al. ${ }^{12}$ These mechanisms are depicted in Scheme 4 and illustrated in the case of the peracetylated glucal 2a. In the first step of these mechanism one electron coming or from the ring oxygen (pathway A) or from the oxygen of the acetate group located on position 3 (pathway B) could be accepted by the nitrosonium cation to give an acetate radical (which in turn could accept an electron from NO to give the acetate anion). Then the delocalized carbocation could undergone nucleophilic displacement reaction by the $\mathrm{N}$-(O-tert-butyldiphenylsilyl)hydroxysulfamide $\mathbf{1}$ with allylic rearrangement leading to the 2,3-unsaturated glycoside $\mathbf{3 a}$ as a mixture of $\alpha$ and $\beta$ anomers, $\alpha$ anomer being predominant.

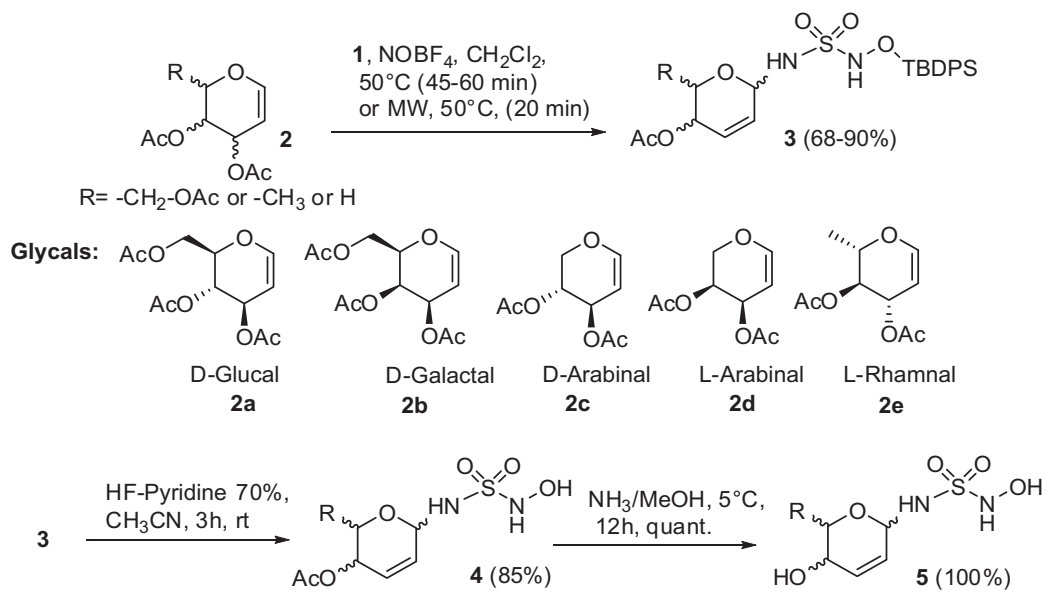

Scheme 3. Synthesis of compound $\mathbf{5}$ via the Ferrier sulfamidoglycosylation. 


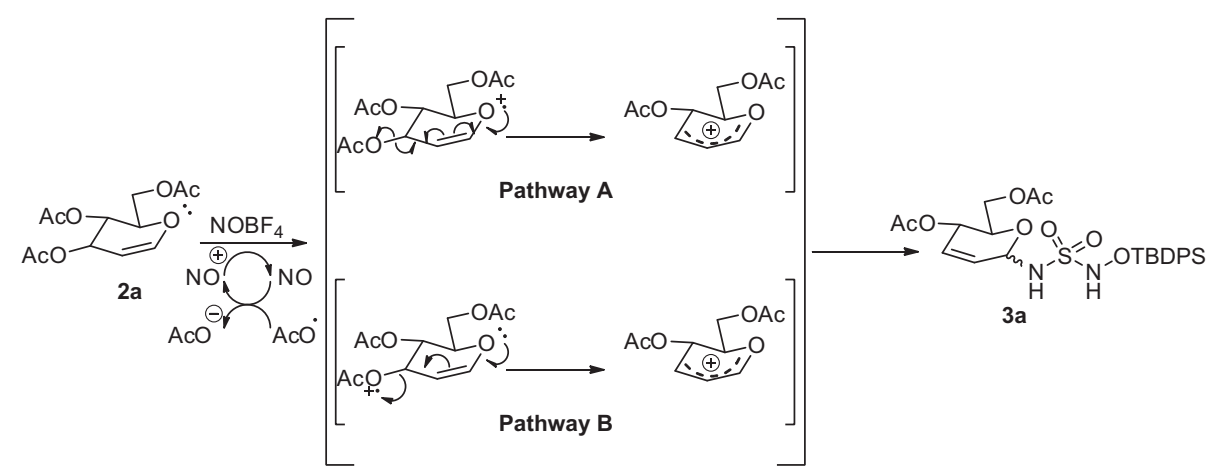

Scheme 4. Proposed mechanisms for the sulfamidoglycosylation reaction/example of Glucal.

Table 2

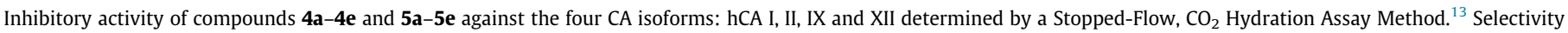
ratios for the inhibition of the tumor-associated (CA IX and XII) over the cytosolic (CA II) isozyme are also reported

\begin{tabular}{|c|c|c|c|c|c|c|}
\hline & \multicolumn{4}{|c|}{$K_{\mathrm{I}}^{\mathrm{a}}(\mathrm{nM})$} & \multicolumn{2}{|c|}{ Selectivity ratio } \\
\hline & hCA I ${ }^{\mathrm{b}}$ & $\mathrm{hCA} \mathrm{II}^{\mathrm{b}}$ & hCA IX & hCA XII $^{\mathrm{c}}$ & $K_{\mathrm{I}}$ hCA II $/ K_{\mathrm{I}}$ hCA IX & $K_{\mathrm{I}} \mathrm{hCA}$ II $/ K_{\mathrm{I}}$ hCA XII \\
\hline $\mathrm{AAZ}$ & 250 & 12 & 25 & 5.6 & 0.48 & 2.14 \\
\hline $4 a$ & 813 & 94 & 90 & 76 & 1.04 & 1.23 \\
\hline $4 b$ & 752 & 86 & 79 & 207 & 1.08 & 0.41 \\
\hline 4c & $>10,000$ & $>10,000$ & 54 & 86 & $>185$ & $>116$ \\
\hline $4 d$ & $>10,000$ & $>10,000$ & 44 & 81 & $>227$ & $>123$ \\
\hline $4 e$ & $>10,000$ & 94 & 47 & 93 & 2 & 1.01 \\
\hline $5 a$ & $>10,000$ & 91 & 9 & 93 & 10.11 & 0.97 \\
\hline $5 \mathbf{b}$ & 917 & $>10,000$ & 44 & 68 & $>227$ & $>147$ \\
\hline $5 c$ & 927 & $>10,000$ & 8 & 88 & $>1250$ & $>113$ \\
\hline $5 d$ & $>10,000$ & 85 & 9 & 415 & 9.44 & 0.20 \\
\hline $5 e$ & $>10,000$ & $>10,000$ & 8 & 87 & $>1250$ & $>114$ \\
\hline
\end{tabular}

a Errors in the range of $\pm 5-10 \%$ of the reported value from three different determinations.

b Full length, cytosolic isoform.

c Catalytic domain, recombinant enzyme.

\section{Carbonic anhydrase inhibition assays}

The compounds reported here were investigated for the inhibition of four human (h) CA isoforms, involved in crucial physiologic processes in mammals, the cytosolic, widespread hCA I and II, as well as the tumor associated, transmembrane isoforms hCA IX and XII. Inhibition data with compounds $\mathbf{4}$ and $\mathbf{5}$, as well as the sulfonamide in clinical use acetazolamide (as standard compound) are reported in Table 2.

It may be observed that the slow cytosolic isoforms (hCA I) was poorly or not at all inhibited by the compounds reported here. Just $\mathbf{4 a}, \mathbf{4 b}$, and $\mathbf{5 b}, \mathbf{5 c}$ showed inhibition constants around $1000 \mathrm{nM}$, whereas all other derivatives were not inhibitory against hCA I up to $10 \mu \mathrm{M}$. hCA II showed a very unusual inhibition profile with the new derivatives. In fact two peracetylated compounds ( $\mathbf{4 c}$ and $\mathbf{4 d})$ and three deacetylated ones $(\mathbf{5 b}, \mathbf{5 c}$ and $\mathbf{5 e})$ were not inhibitory up to concentrations of $10 \mu \mathrm{M}$, whereas the remaining ones showed medium potency inhibitory action, with $K_{\mathrm{I}} \mathrm{S}$ in the range of 85-94 nM. Thus, in this case the nature of the glycal strongly influences activity but in manner difficult to rationalize considering both the acetylated as well as the deacetylated series.

The tumor associated hCA IX on the other hand was effectively inhibited by all the new compounds, with the acetylated derivatives 4 being slightly less effective ( $K_{\mathrm{I}} \mathrm{S}$ in the range of 44-90 nM) than the deacetylated ones, $\mathbf{5}$, which had $K_{\mathrm{I}} \mathrm{S}$ in the range of 8$44 \mathrm{nM}$. Generally the nature of the glycal was not very important for the inhibitory power except galactal which led to a derivative around one order of magnitude less potent compared to the other sugar derivatives investigated here. hCA XII was moderately inhibited by both compounds 4 and $\mathbf{5}$ reported here, with $K_{\mathrm{I}} \mathrm{S}$ in the range of 68-415 nM, with two compounds in each series (4b and 5d, respectively) showing the weakest inhibition $\left(K_{\mathrm{I}} \mathrm{S}\right.$ of 207 and $415 \mathrm{nM}$, respectively) whereas the remaining ones showed a quite compact behavior of medium potency inhibitor $\left(K_{\mathrm{I}} \mathrm{S}\right.$ in the range of 68-93 nM).

Some of the new compounds reported here, such as $\mathbf{4 c}, \mathbf{4 d}, \mathbf{5 b}$, $\mathbf{5 c}$ and $\mathbf{5 e}$ showed profiles of significant tumor-associated CAs selective inhibitors, with selectivity ratios of $>100$ for inhibiting hCA IX/XII over hCA I/II.

\section{Conclusion}

In conclusion, our methodology allowed the preparation of hydroxysulfamide glycosides from glycals in good yields and with a good alpha stereoselectivity. We have used an effective sulfamidoglycosylation method using $\mathrm{NOBF}_{4}$ as a catalyst in dichloromethane. Studies on the inhibitory activity against the two cytosolic CA isoforms: hCA I, II, and the two tumor associated, membrane isoforms hCA IX and XII demonstrated the importance of the sugar scaffold in the design of powerful and selective hCA IX/hCA XII inhibitors with potential in antitumor therapy, although the structure-activity relationship in this small series of inhibitors is not at all straightforward.

\section{Experimental section}

\subsection{General methods}

Microwaves synthesis was carried out on CEM Discover Microwaves of CEM Corporation. The thin layer chromatographies (TLC) 
were performed on silica plates Merck 60 F354 aluminum. Reactions were monitored by TLC by using alumina plates coated with silica gel and visualized either by using UV light or by charring with of $10 \%$ sulfuric acid in ethanol solution. Column chromatography was performed on silica gel $60 \AA$, particle size: $35-70$ mesh. ${ }^{1} \mathrm{H}$ and ${ }^{13} \mathrm{C}$ NMR spectra were recorded on Brucker-400 instrument using the residual solvent signals as an internal reference. Highresolution mass spectra (HRMS) were obtained from an ESI-TOFMS spectrometer (SYNAPT G2-S of Waters).

\subsection{Microwave irradiation experiments}

All microwave experiments were performed with CEM Discover Synthesizer possessing (Sp). On this device, the temperature is controlled via an optical fiber directly inside the reactor, providing greater accuracy in the measurements. Experiments were carried out in standard microwaves process vials $10 \mathrm{ml}$ capacity (filled with $7 \mathrm{ml}$ max). The specifications used are pressure: 17 bars; power: $150-200 \mathrm{~W}$; temperature: $50^{\circ} \mathrm{C}$ and power max: off.

\subsection{Synthesis}

\subsection{1. $\mathbf{N}$-(O-tert-Butyldiphenylsilyl)hydroxysulfamide (1)}

Triethylamine $(5 \mathrm{~mL}, 37 \mathrm{mmol})$ was added dropwise to a suspension of hydroxylamine hydrochloride ( $1 \mathrm{~g}, 14.4 \mathrm{mmol}$ ) in anhydrous $\mathrm{CH}_{2} \mathrm{Cl}_{2}(20 \mathrm{~mL})$ under $\mathrm{N}_{2}$ at $0{ }^{\circ} \mathrm{C}$. The mixture was stirred at room temperature for $2 \mathrm{~h}$. then cooled to $0^{\circ} \mathrm{C}$. A solution of tertbutyldiphenylsilyl chloride TBDPSCl $(1.80 \mathrm{~g}, 12 \mathrm{mmol})$ in anhydrous $\mathrm{CH}_{2} \mathrm{Cl}_{2}(10 \mathrm{~mL})$ was then slowly added at $0{ }^{\circ} \mathrm{C}$. The reaction mixture was stirred at room temperature for $20 \mathrm{~h}$. In a separate flask, tert-butanol $(1.14 \mathrm{~mL}, 12 \mathrm{mmol})$ was added dropwise to a solution of chlorosulfonylisocyanate $(1.02 \mathrm{~mL}, 12 \mathrm{mmol})$ in anhydrous $\mathrm{CH}_{2} \mathrm{Cl}_{2}(30 \mathrm{~mL})$ under $\mathrm{N}_{2}$ at $0{ }^{\circ} \mathrm{C}$ and stirred at $0{ }^{\circ} \mathrm{C}$ for 40 min to give the tert-butoxycarbamoylsulfamoyl chloride. Triethylamine ( $5 \mathrm{~mL}, 37 \mathrm{mmol}$ ) was added to the solution of protected hydroxylamine at $0{ }^{\circ} \mathrm{C}$ and the solution containing the tert-butoxycarbamoylsulfamoyl chloride was added dropwise via a syringe. The reaction mixture was warmed to rt and stirred for $18 \mathrm{~h}$. The solvent was removed under reduced pressure. The residue was dissolved in EtOAc $(100 \mathrm{~mL})$ and washed with water $(10 \mathrm{~mL}), 0.1 \mathrm{M}$ $\mathrm{HCl}(3 \times 10 \mathrm{~mL})$, satd $\mathrm{NaHCO}_{3}(3 \times 10 \mathrm{~mL})$, brine $(15 \mathrm{~mL})$. The organic layer was finally dried over anhydrous sodium sulfate and concentrated under reduced pressure. The residue obtained was then dissolved and stirred at $0{ }^{\circ} \mathrm{C}$ in a solution of $10 \%$ TFA- $\mathrm{CH}_{2} \mathrm{Cl}_{2}$. The reaction was monitored by TLC until the complete disappearance of starting material. The solvent was removed under reduced pressure to give the expected compound 1 in 78\% yield.

$\mathrm{Mp}=110{ }^{\circ} \mathrm{C} ; \quad R_{f}=0.30 \quad\left(100 \% \quad \mathrm{CH}_{2} \mathrm{Cl}_{2}\right) ;{ }^{1} \mathrm{H} \quad \mathrm{NMR}(400 \mathrm{MHz}$, DMSO-d $\left.d_{6}\right) \delta 8.91(\mathrm{~s}, 1 \mathrm{H}, \mathrm{NH}), 7.70-7.79\left(\mathrm{~m}, 4 \mathrm{H}, 4 \times \mathrm{CH}_{\text {ortho }}\right), 7.43-$ $7.48\left(\mathrm{~m}, 2 \mathrm{H}, 2 \times \mathrm{CH}_{\text {para }}\right), 7.36-7.43\left(\mathrm{~m}, 4 \mathrm{H}, 4 \times \mathrm{CH}_{\text {meta }}\right), 6.95(\mathrm{~s}$, $\left.2 \mathrm{H}, \mathrm{NH}_{2}\right), 1.07\left(\mathrm{~s}, 9 \mathrm{H}, \mathrm{C}\left(\mathrm{CH}_{3}\right)_{3}\right) .{ }^{13} \mathrm{C}$ NMR (101 MHz, DMSO-d $\left.\mathrm{d}_{6}\right) \delta$ $135.5\left(\mathrm{CH}_{\text {ortho }}\right), 132.4$ ( $\left.\mathrm{CSi}\right), 129.7\left(\mathrm{CH}_{\text {para }}\right), 127.4\left(\mathrm{CH}_{\text {meta }}\right), 26.8$ $\left.\left.\left(\mathrm{C}_{(\mathrm{CH}}\right)_{3}\right), 19.1\left(\mathrm{C} \mathrm{CH}_{3}\right)_{3}\right)$. MS ESI $m / z 351.15[\mathrm{M}+\mathrm{H}]^{+}$.

\subsubsection{General procedure for the synthesis of compound 3}

To a solution of peracetylated glycal $(1 \mathrm{mmol})$ and $\mathrm{N}$-(O-tertbutyldiphenylsilyl)hydroxysulfamide $(1.1 \mathrm{mmol}, 1.1$ equiv) in $5 \mathrm{ml}$ of dry $\mathrm{CH}_{2} \mathrm{Cl}_{2}$ was added $\mathrm{NOBF}_{4}(0.05 \mathrm{mmol}, 0.05$ equiv). The mixture was then, or stirred under reflux $\left(50^{\circ} \mathrm{C}\right)$ or stirred under microwaves irradiations until completion of the reaction. The reaction mixture was concentrated under reduced pressure and the crude product was purified on silica gel using diethyl ether and pentane as eluent (3a, 3b and $\mathbf{3 e}: \mathrm{Et}_{2} \mathrm{O} /$ pentane $5: 5$; $\mathbf{3 c}$ and $\mathbf{3 d}$ $\mathrm{Et}_{2} \mathrm{O} /$ pentane 6:4). Compound 3 are obtained as a mixture of $\alpha / \beta$ isomers.
5.3.2.1. Compound 3a. We introduced $0.272 \mathrm{~g}$ of $\mathbf{2 a}$ and we obtained $0.383 \mathrm{~g}$ (yield $\Delta: 68 \%$ ) and $0.394 \mathrm{~g}$ (MW: 70\%). HRMS calcd for $\mathrm{C}_{26} \mathrm{H}_{35} \mathrm{~N}_{2} \mathrm{O}_{8} \mathrm{SSi}[\mathrm{M}+\mathrm{H}]^{+}$: 563.1883, found 563.1880 ( $-0.3 \mathrm{mDa})$.

5.3.2.1.1. N-[1-(4,6-Di-O-acetyl-2,3-dideoxy- $\alpha$-D-erythro-hex-2eno-pyranosyl)]-N-(O-tert-butyldiphenylsilyl)sulfamide (3a, anomer $\alpha, 60 \%) . \quad{ }^{1} \mathrm{H}$ NMR (400 MHz, DMSO-d $\left.d_{6}\right) \delta 9.25$ (s, 1H, NHOSi), 9.01 (d, $1 \mathrm{H}, J=8.6 \mathrm{~Hz}, \mathrm{NHCH}), 7.74-7.66\left(\mathrm{~m}, 4 \mathrm{H}, 4 \times \mathrm{CH}_{\text {ortho }}\right), 7.50-7.44$ ( $\left.\mathrm{m}, 2 \mathrm{H}, 2 \times \mathrm{CH}_{\text {para }}\right), 7.43-7.38\left(\mathrm{~m}, 4 \mathrm{H}, 4 \times \mathrm{CH}_{\text {meta }}\right), 5.90$ (td, $J=10.2$, $1.6 \mathrm{~Hz}, 1 \mathrm{H}, \mathrm{H}-3$ ), 5.82 (ddd, $J=10.2,2.9,2.1 \mathrm{~Hz}, 1 \mathrm{H}, \mathrm{H}-2$ ), 5.37 (ddd, $J=8.6,2.9,1.6 \mathrm{~Hz} 1 \mathrm{H}, \mathrm{H}-1), 5.20-5.16(\mathrm{~m}, 1 \mathrm{H}, \mathrm{H}-4), 4.15(\mathrm{dd}$ $J=11.6,2.5 \mathrm{~Hz}, 1 \mathrm{H}, \mathrm{H}-6 \mathrm{a}), 4.11-4.09(\mathrm{~m}, 1 \mathrm{H}, \mathrm{H}-5), 4.03(\mathrm{dd}$, $J=11.6,4.3 \mathrm{~Hz}, 1 \mathrm{H}, \mathrm{H}-6 \mathrm{~b}), 2.06\left(\mathrm{~s}, 3 \mathrm{H}, \mathrm{CH}_{3} \mathrm{COO}\right), 1.98(\mathrm{~s}, 3 \mathrm{H}$, $\left.\mathrm{CH}_{3} \mathrm{COO}\right), 1.06\left(\mathrm{~s}, 9 \mathrm{H}, 3 \times \mathrm{CH}_{3}\right) .{ }^{13} \mathrm{C}$ NMR (101 MHz, DMSO- $\left.d_{6}\right) \delta$ $169.9(\mathrm{C}=\mathrm{O}), 169.6(\mathrm{C}=\mathrm{O}), 135.5\left(\mathrm{CH}_{\text {ortho }}\right), 131.9$ (CSi), 130.2 $\left(\mathrm{CH}_{\text {para }}\right), 128.4(\mathrm{C}-3), 127.6(\mathrm{C}-2), 127.3\left(\mathrm{CH}_{\text {meta }}\right), 76.2(\mathrm{C}-1), 66.8$ (C-5), 64.1 (C-4), $62.3(\mathrm{C}-6), 26.61\left(\mathrm{C}\left(\mathrm{CH}_{3}\right)_{3}\right), 20.59\left(\mathrm{CH}_{3} \mathrm{COO}\right)$, $20.29\left(\mathrm{CH}_{3} \mathrm{COO}\right), 18.93\left(\mathrm{C}\left(\mathrm{CH}_{3}\right)_{3}\right)$.

5.3.2.1.2. N-[1-(4,6-Di-O-acetyl-2,3-dideoxy- $\beta$-D-threo-hex-2-enopyranosyl)]-N-(O-tert-butyldiphenylsilyl)sulfamide (3a, anomer $\beta$, 40\%). $\quad{ }^{1} \mathrm{H}$ NMR (400 MHz, DMSO- $\left.d_{6}\right) \delta 9.18$ (s, 1H, NHOSi), 8.88 $(\mathrm{d}, J=8.6 \mathrm{~Hz}, 1 \mathrm{H}, \mathrm{NHCH}), 7.74-7.66\left(\mathrm{~m}, 4 \mathrm{H}, 4 \times \mathrm{CH}_{\text {ortho }}\right), 7.50-$ $7.44\left(\mathrm{~m}, 2 \mathrm{H}, 2 \times \mathrm{CH}_{\text {para }}\right), 7.43-7.38\left(\mathrm{~m}, 4 \mathrm{H}, 4 \times \mathrm{CH}_{\text {meta }}\right), 5.87(\mathrm{td}$, $J=10.2,1.6 \mathrm{~Hz}, 1 \mathrm{H}, \mathrm{H}-3), 5.86(\mathrm{td}, J=10.2,1.3 \mathrm{~Hz}, 1 \mathrm{H}, \mathrm{H}-2), 5.44-$ $5.39(\mathrm{~m}, 1 \mathrm{H}, \mathrm{H}-1), 5.24-5.20(\mathrm{~m}, 1 \mathrm{H}, \mathrm{H}-4), 4.15(\mathrm{dd}, J=11.6$, $2.5 \mathrm{~Hz}, 1 \mathrm{H}, \mathrm{H}-6 \mathrm{a}$ ), 4.05 (dd, $J=11.6,4.3 \mathrm{~Hz}, 1 \mathrm{H}, \mathrm{H}-6 \mathrm{~b}$ ), 3.86 (ddd, $J=8.25,4.74,3.24 \mathrm{~Hz}, 1 \mathrm{H}, \mathrm{H}-5), 2.04\left(\mathrm{~s}, 3 \mathrm{H}, \mathrm{CH}_{3} \mathrm{COO}\right), 1.97(\mathrm{~s}, 3 \mathrm{H}$, $\mathrm{CH}_{3} \mathrm{COO}$ ), 1.07 (s, 9H, $\left.3 \times \mathrm{CH}_{3}\right) .{ }^{13} \mathrm{C}$ NMR $\left(101 \mathrm{MHz}\right.$, DMSO-d $\left.d_{6}\right) \delta$ $169.8(\mathrm{C}=\mathrm{O}), 169.6(\mathrm{C}=\mathrm{O}), 135.5\left(\mathrm{CH}_{\text {ortho }}\right), 132.0$ (CSi), 130.2 $\left(\mathrm{CH}_{\text {para }}\right), 129.8(\mathrm{C}-3), 127.8(\mathrm{C}-2), 127.3\left(\mathrm{CH}_{\text {meta }}\right), 78.8(\mathrm{C}-1), 73.2$ (C-5), 63.9 (C-4), $62.6(\mathrm{C}-6), 26.61\left(\mathrm{C}\left(\mathrm{CH}_{3}\right)_{3}\right), 20.59\left(\mathrm{CH}_{3} \mathrm{COO}\right)$, $20.31\left(\mathrm{CH}_{3} \mathrm{COO}\right), 18.87\left(\mathrm{C}\left(\mathrm{CH}_{3}\right)_{3}\right)$.

5.3.2.2. Compound 3b. We introduced $0.272 \mathrm{~g}$ of $\mathbf{2 b}$ and we obtained $0.394 \mathrm{~g}$ (yield $\Delta: 70 \%$ ) and $0.394 \mathrm{~g}$ (MW: 70\%). HRMS calcd for $\mathrm{C}_{26} \mathrm{H}_{35} \mathrm{~N}_{2} \mathrm{O}_{8} \mathrm{SSi}[\mathrm{M}+\mathrm{H}]^{+}$: 563.1883, found 563.1880 (-0.3 mDa).

5.3.2.2.1. $N$-[1-(4,6-Di-O-acetyl-2,3-dideoxy- $\alpha$-D-threo-hex-2-enopyranosyl)]-N-(O-tert-butyldiphenylsilyl)sulfamide (3b, anomer $\alpha$, 75\%). ${ }^{1} \mathrm{H}$ NMR (400 MHz, DMSO-d $\left.d_{6}\right) \delta 9.29$ (s, 1H, NHOSi), 8.90 $(\mathrm{d}, J=9.0 \mathrm{~Hz}, 1 \mathrm{H}, \mathrm{NHCH}), 7.75-7.65\left(\mathrm{~m}, 4 \mathrm{H}, 4 \times \mathrm{CH}_{\text {ortho }}\right), 7.50-$ $7.44\left(\mathrm{~m}, 2 \mathrm{H}, 2 \times \mathrm{CH}_{\text {para }}\right.$ ), 7.44-7.37 ( $\left.\mathrm{m}, 4 \mathrm{H}, 4 \times \mathrm{CH}_{\text {méta }}\right), 6.06$ (ddd, $J=9.9,5.4,1.4 \mathrm{~Hz}, 1 \mathrm{H}, \mathrm{H}-3), 6.02(\mathrm{dd}, J=9.9,3.2 \mathrm{~Hz}, 1 \mathrm{H}, \mathrm{H}-2$ ), 5.39 (ddd, $J=9.0,3.0,1.3 \mathrm{~Hz}, 1 \mathrm{H}, \mathrm{H}-1$ ), 4.96 (dd, $1 \mathrm{H}, \mathrm{H}-4), 4.40$ (dd, $J=6.5,2.5 \mathrm{~Hz}, 1 \mathrm{H}, \mathrm{H}-5), 4.14$ (dd, $J=11.2,6.5 \mathrm{~Hz}, 1 \mathrm{H}, \mathrm{H}-6 \mathrm{a}$ ), 4.00 (dd, $J=11.2,6.5 \mathrm{~Hz}, 1 \mathrm{H}, \mathrm{H}-6 \mathrm{~b}$ ), 2.01 (s, 3H, $\mathrm{CH}_{3} \mathrm{COO}$ ), 1.93 (s, $\left.3 \mathrm{H}, \mathrm{CH}_{3} \mathrm{COO}\right), 1.07$ (s, 9H, $\left.3 \times \mathrm{CH}_{3}\right) .{ }^{13} \mathrm{C}$ NMR $\left(101 \mathrm{MHz}, \mathrm{DMSO}-d_{6}\right)$ $\delta 170.2(\mathrm{C}=\mathrm{O}), 170.1(\mathrm{C}=\mathrm{O}), 135.9\left(\mathrm{CH}_{\text {ortho }}\right), 132.3(\mathrm{CSi}), 130.8(\mathrm{C}-$ 2), $130.2\left(\mathrm{CH}_{\text {para }}\right), 127.8\left(\mathrm{CH}_{\text {meta }}\right), 124.9(\mathrm{C}-3), 76.3(\mathrm{C}-1), 67.1$ (C-

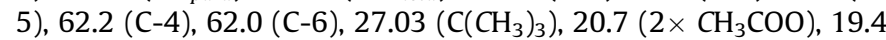
$\left(\mathrm{C}\left(\mathrm{CH}_{3}\right)_{3}\right)$.

5.3.2.2.2. $\quad N$-[1-(4,6-Di-O-acetyl-2,3-dideoxy- $\beta$-D-erythro-hex-2eno-pyranosyl)]-N-(O-tert-butyldiphenylsilyl)sulfamide (3b, anomer $\beta, 25 \%) .{ }^{1} \mathrm{H}$ NMR (400 MHz, DMSO- $\left.d_{6}\right) \delta 9.14$ (s, $\left.1 \mathrm{H}, \mathrm{NHOSi}\right)$, $9.00(\mathrm{~d}, J=9.4 \mathrm{~Hz}, 1 \mathrm{H}, \mathrm{NHCH}), 7.75-7.65\left(\mathrm{~m}, 4 \mathrm{H}, 4 \times \mathrm{CH}_{\text {ortho }}\right)$, 7.50-7.44 (m, 2H, 4× $\left.\mathrm{CH}_{\text {para }}\right), 7.44-7.37\left(\mathrm{~m}, 4 \mathrm{H}, 4 \times \mathrm{CH}_{\text {meta }}\right)$, 6.04 (ddd, $J=10.0,5.0,1.8,1 \mathrm{H}, \mathrm{H}-3$ ), 5.99 (br d, $J=10.0 \mathrm{~Hz}, 1 \mathrm{H}$, $\mathrm{H}-2), 5.31$ (d, $J=8.2 \mathrm{~Hz}, 1 \mathrm{H}, \mathrm{H}-1), 5.05(\mathrm{td}, J=5.0,1.8 \mathrm{~Hz}$, $1 \mathrm{H}, \mathrm{H}-4), 4.09-4.11$ (m, 2H, H-6a, H-6b), 4.03 (dt, $J=6.2,2.4 \mathrm{~Hz}$, $1 \mathrm{H}, \mathrm{H}-5), 2.02\left(\mathrm{~s}, 3 \mathrm{H}, \mathrm{CH}_{3} \mathrm{COO}\right), 1.97\left(\mathrm{~s}, 3 \mathrm{H}, \mathrm{CH}_{3} \mathrm{COO}\right), 1.07$ (s, $\left.9 \mathrm{H}, 3 \times \mathrm{CH}_{3}\right) .{ }^{13} \mathrm{C}$ NMR $\left(101 \mathrm{MHz}\right.$, DMSO-d $\left.d_{6}\right) \delta 170.4(\mathrm{C}=\mathrm{O})$, 170.3 (C=O), 135.9 ( $\left(\mathrm{CH}_{\text {ortho }}\right), 132.3$ (CSi), 130.8 (C-2), 130.3 $\left(\mathrm{CH}_{\text {para }}\right), 127.8\left(\mathrm{CH}_{\text {meta }}\right), 124.9(\mathrm{C}-3), 79.4(\mathrm{C}-1), 72.3(\mathrm{C}-5), 63.0$ (C-4), 62.5 (C-6), $26.99\left(\mathrm{C}\left(\mathrm{CH}_{3}\right)_{3}\right), 20.81\left(\mathrm{CH}_{3} \mathrm{COO}\right), 20.76\left(\mathrm{CH}_{3-}\right.$

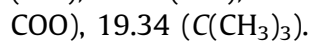


5.3.2.3. Compound 3c. We introduced $0.200 \mathrm{~g}$ of $\mathbf{2 c}$ and we obtained $0.431 \mathrm{~g}$ (yield $\Delta: 88 \%$ ) and $0.441 \mathrm{~g}$ (MW: 90\%). HRMS calcd for $\mathrm{C}_{23} \mathrm{H}_{31} \mathrm{~N}_{2} \mathrm{O}_{6} \mathrm{SSi}[\mathrm{M}+\mathrm{H}]^{+}:$491.1672, found 491.1675 (0.3 $\mathrm{mDa})$.

5.3.2.3.1. N-[1-(4-O-Acetyl-5-anhydro-2,3-dideoxy- $\alpha$-D-erythropent-2-enopyranosyl)]-N-(O-tert-butyldiphenylsilyl)sulfamide (3c, anomer $\alpha, 60 \%) .{ }^{1} \mathrm{H}$ NMR (400 MHz, DMSO- $\left.d_{6}\right) \delta 9.13(\mathrm{~s}, 1 \mathrm{H}$, NHOSi), $8.86(\mathrm{~d}, J=9.0 \mathrm{~Hz}, 1 \mathrm{H}, \mathrm{NHCH}), 7.74-7.67(\mathrm{~m}, 4 \mathrm{H}, 4 \times$ $\left.\mathrm{CH}_{\text {ortho }}\right), 7.50-7.44\left(\mathrm{~m}, 2 \mathrm{H}, 2 \times \mathrm{CH}_{\text {para }}\right), 7.44-7.37(\mathrm{~m}, 4 \mathrm{H}, 4 \times$ $\left.\mathrm{CH}_{\text {meta }}\right), 6.04$ (tdd, $\left.J=9.9,4.9,1.3 \mathrm{~Hz}, 1 \mathrm{H}, \mathrm{H}-3\right), 5.99-5.96(\mathrm{~m}, 1 \mathrm{H}$, $\mathrm{H}-2)$, 5.35-5.31 (m, 1H, H-1), 4.90-4.84 (m, 1H, H-4), 4.18 (dd, $J=13.1,1.3 \mathrm{~Hz}, 1 \mathrm{H}, \mathrm{H}-5 \mathrm{a}), 3.74(\mathrm{dd}, J=13.1,1.3 \mathrm{~Hz}, 1 \mathrm{H}, \mathrm{H}-5 \mathrm{~b})$, 2.03 (s, 3H, CH $\left.\mathrm{CHOO}_{3}\right), 1.07\left(\mathrm{~s}, 9 \mathrm{H}, 3 \times \mathrm{CH}_{3}\right) .{ }^{13} \mathrm{C} \mathrm{NMR}(101 \mathrm{MHz}$, DMSO- $\left.d_{6}\right) \delta 170.2(\mathrm{C}=\mathrm{O}), 135.9\left(\mathrm{CH}_{\text {ortho }}\right), 132.3(\mathrm{CSi}), 130.9(\mathrm{C}-2)$, $130.2\left(\mathrm{CH}_{\text {para }}\right), 127.79\left(\mathrm{CH}_{\text {meta }}\right), 125.1(\mathrm{C}-3), 75.5(\mathrm{C}-1), 63.2(\mathrm{C}-4)$, $61.8(\mathrm{C}-5), 26.95\left(\mathrm{C}\left(\mathrm{CH}_{3}\right)_{3}\right), 21.04\left(\mathrm{CH}_{3} \mathrm{COO}\right), 19.3\left(\mathrm{C}_{\left.\left(\mathrm{CH}_{3}\right)_{3}\right) .}\right.$

5.3.2.3.2. $\mathrm{N}$-[1-(4-O-Acetyl-5-anhydro-2,3-dideoxy- $\beta$-D-threo-pent2-enopyranosyl)]-N-(O-tert-butyldiphenylsilyl)sulfamide (3c, anomer $\beta, 40 \%) . \quad{ }^{1} \mathrm{H}$ NMR (400 MHz, DMSO- $\left.d_{6}\right) \delta 9.15$ (s, $\left.1 \mathrm{H}, \mathrm{NHOSi}\right), 8.96$ (d, $J=9.0 \mathrm{~Hz}, 1 \mathrm{H}, \mathrm{NHCH}$ ), 7.74-7.67 ( $\mathrm{m}, 4 \mathrm{H}, 4 \times \mathrm{CH}_{\text {ortho }}$ ), 7.50-7.44 $\left(\mathrm{m}, 2 \mathrm{H}, 2 \times \mathrm{CH}_{\text {para }}\right), 7.44-7.37\left(\mathrm{~m}, 4 \mathrm{H}, 4 \times \mathrm{CH}_{\text {meta }}\right), 6.01-5.99(\mathrm{~m}$, $1 \mathrm{H}, \mathrm{H}-3$ ), 5.88 (ddd, $J=10.1,2.2,1.3 \mathrm{~Hz}, 1 \mathrm{H}, \mathrm{H}-2$ ), 5.26 (dd, $J=9.0$, $2.0 \mathrm{~Hz}, 1 \mathrm{H}, \mathrm{H}-1$ ), 5.10-5.04 (m, 1H, H-4), 3.81 (dd, $J=11.8,4.9 \mathrm{~Hz}$, $1 \mathrm{H}, \mathrm{H}-5 \mathrm{a}$ ), 3.80 (dd, $J=11.8,5.8 \mathrm{~Hz}, 1 \mathrm{H}, \mathrm{H}-5 \mathrm{~b}), 2.03\left(\mathrm{~s}, 3 \mathrm{H}, \mathrm{CH}_{3} \mathrm{COO}\right.$ ), $1.07\left(\mathrm{~s}, 9 \mathrm{H}, 3 \times \mathrm{CH}_{3}\right) .{ }^{13} \mathrm{C}$ NMR (101 MHz, DMSO-d $\left.{ }_{6}\right) \delta 170.3(\mathrm{C}=\mathrm{O})$, $135.9\left(\mathrm{CH}_{\text {ortho }}\right) 132.3(\mathrm{CSi}), 130.6(\mathrm{C}-2), 130.2\left(\mathrm{CH}_{\text {para }}\right), 127.8(\mathrm{C}-3)$,

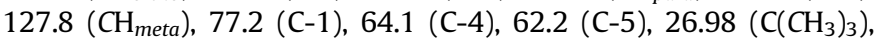
$21.0\left(\mathrm{CH}_{3} \mathrm{COO}\right), 19.3\left(\mathrm{C}\left(\mathrm{CH}_{3}\right)_{3}\right)$.

5.3.2.4. Compound 3d. We introduced $0.200 \mathrm{~g}$ of $2 \mathrm{~d}$ and we obtained $0.421 \mathrm{~g}$ (yield $\Delta: 86 \%$ ) and $0.441 \mathrm{~g}$ (MW: 90\%). HRMS calcd for $\mathrm{C}_{23} \mathrm{H}_{31} \mathrm{~N}_{2} \mathrm{O}_{6} \mathrm{SSi}[\mathrm{M}+\mathrm{H}]{ }^{+}$: 491.1672, found 491.1672 (0.0 mDa).

5.3.2.4.1. $\quad N$-[1-(4-O-Acetyl-5-anhydro-2,3-dideoxy- $\alpha$-L-erythropent-2-enopyranosyl)]-N-(O-tert-butyldiphenylsilyl)sulfamide (3d, anomer $\alpha, 60 \%) .{ }^{1} \mathrm{H}$ NMR (400 MHz, DMSO- $\left.d_{6}\right) \delta 9.13(\mathrm{~s}, 1 \mathrm{H}$, NHOSi), $8.86(\mathrm{~d}, J=9.0 \mathrm{~Hz}, 1 \mathrm{H}, \mathrm{NHCH}), 7.75-7.67(\mathrm{~m}, 4 \mathrm{H}, 4 \times$ $\left.\mathrm{CH}_{\text {ortho }}\right), 7.50-7.44\left(\mathrm{~m}, 2 \mathrm{H}, 2 \times \mathrm{CH}_{\text {para }}\right), 7.44-7.38(\mathrm{~m}, 4 \mathrm{H}, 4 \times$ $\left.\mathrm{CH}_{\text {meta }}\right), 6.04$ (tdd, $\left.J=10.1,4.9,1.4 \mathrm{~Hz}, 1 \mathrm{H}, \mathrm{H}-3\right), 6.02-5.99(\mathrm{~m}$, $1 \mathrm{H}, \mathrm{H}-2$ ), 5.33 (dd, $J=9.0,1.8 \mathrm{~Hz}, 1 \mathrm{H}, \mathrm{H}-1), 4.89-4.84(\mathrm{~m}, 1 \mathrm{H}, \mathrm{H}-$ 4), 4.18 (dd, $J=13.2,2.9 \mathrm{~Hz}, 1 \mathrm{H}, \mathrm{H}-5 \mathrm{a}$ ), 3.74 (dd, $J=13.2,1.4 \mathrm{~Hz}$, $1 \mathrm{H}, \mathrm{H}-5 \mathrm{~b}), 2.03$ (s, 3H, $\left.\mathrm{CH}_{3} \mathrm{COO}\right), 1.07$ (s, 9H, 3× $\left.\mathrm{CH}_{3}\right) .{ }^{13} \mathrm{C} \mathrm{NMR}$ $\left(101 \mathrm{MHz}\right.$, DMSO-d $\left.\mathrm{d}_{6}\right) \delta 170.3(\mathrm{C}=\mathrm{O}), 135.87\left(\mathrm{CH}_{\text {ortho }}\right), 132.26$ (CSi), 130.9 (C-2), $130.2\left(\mathrm{CH}_{\text {para }}\right), 127.80\left(\mathrm{CH}_{\text {meta }}\right), 125.1(\mathrm{C}-3)$,

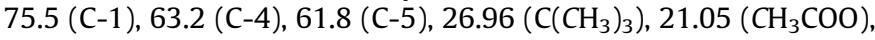
$19.30\left(\mathrm{C}\left(\mathrm{CH}_{3}\right)_{3}\right)$.

5.3.2.4.2. $N$-[1-(4-O-Acetyl-5-anhydro-2,3-dideoxy- $\beta$-L-thréo-pent2-enopyranosyl)]-N-(O-tert-butyldiphenylsilyl)sulfamide (3d, anomer $\beta, 40 \%) . \quad{ }^{1} \mathrm{H}$ NMR (400 MHz, DMSO- $\left.d_{6}\right) \delta 9.15$ (s, $\left.1 \mathrm{H}, \mathrm{NHOSi}\right), 8.95$ (d, $J=9.0 \mathrm{~Hz}, 1 \mathrm{H}, \mathrm{NHCH}), 7.75-7.67\left(\left(\mathrm{~m}, 4 \mathrm{H}, 4 \times \mathrm{CH}_{\text {ortho }}\right), 7.50-7.44\right.$ (m, $\left.2 \mathrm{H}, 2 \times \mathrm{CH}_{\text {para }}\right), 7.44-7.38\left(\mathrm{~m}, 4 \mathrm{H}, 4 \times \mathrm{CH}_{\text {meta }}\right), 5.99-5.97(\mathrm{~m}$, $1 \mathrm{H}, \mathrm{H}-3$ ), 5.90 (ddd, $J=10.1,2.1,1.3 \mathrm{~Hz}, 1 \mathrm{H}, \mathrm{H}-2$ ), 5.26 (dd, $J=9.0$, $1.9 \mathrm{~Hz}, 1 \mathrm{H}, \mathrm{H}-1), 5.12-5.03(\mathrm{~m}, 1 \mathrm{H}, \mathrm{H}-4), 3.82(\mathrm{dd}, J=11.7,6.0 \mathrm{~Hz}$, $1 \mathrm{H}, \mathrm{H}-5 \mathrm{a}), 3.79$ (dd, $J=11.7,4.7 \mathrm{~Hz}, 1 \mathrm{H}, \mathrm{H}-5 \mathrm{~b}), 2.03$ (s, 2H), 2.03 (s, $\left.3 \mathrm{H}, \mathrm{CH}_{3} \mathrm{COO}\right), 1.08\left(\mathrm{~s}, 9 \mathrm{H}, 3 \times \mathrm{CH}_{3}\right) .{ }^{13} \mathrm{C}$ NMR (101 MHz, DMSO- $\left.d_{6}\right)$ $\delta 170.2(\mathrm{C}=\mathrm{O}), 135.85\left(\mathrm{CH}_{\text {ortho }}\right), 132.3(\mathrm{CSi}), 130.6(\mathrm{C}-2), 130.1$ $\left(\mathrm{CH}_{\text {para }}\right), 127.82$ (C-3), $127.80\left(\mathrm{CH}_{\text {meta }}\right), 77.2(\mathrm{C}-1), 64.1$ (C-4), 62.2 (C-5), $26.98\left(\mathrm{C}\left(\mathrm{CH}_{3}\right)_{3}\right), 21.00\left(\mathrm{CH}_{3} \mathrm{COO}\right), 19.30\left(\mathrm{C}\left(\mathrm{CH}_{3}\right)_{3}\right)$.

5.3.2.5. Compound 3e. We introduced $0.214 \mathrm{~g}$ of $\mathbf{2 e}$ and we obtained $0.444 \mathrm{~g}$ (yield $\Delta: 88 \%$ ) and $0.454 \mathrm{~g}$ (MW: 90\%). HRMS calcd for $\mathrm{C}_{24} \mathrm{H}_{33} \mathrm{~N}_{2} \mathrm{O}_{6} \mathrm{SSi}[\mathrm{M}+\mathrm{H}]^{+}$: 505.1829 found 505.1826 $(-0.3 \mathrm{mDa})$.

5.3.2.5.1. N-[1-(4-O-Acetyl-6-anhydro-2,3-dideoxy- $\alpha$-L-threo-hex2-enopyranosyl)]- $\mathrm{N}$-(O-tert-butyldiphenylsilyl)sulfamide (3e, anomer $\alpha, 84 \%) . \quad{ }^{1} \mathrm{H}$ NMR (400 MHz, DMSO- $\left.d_{6}\right) \delta 9.16$ (s, $\left.1 \mathrm{H}, \mathrm{NHOSi}\right), 8.78$ (d, $J=9.2 \mathrm{~Hz}, 1 \mathrm{H}, \mathrm{NHCH}), 7.74-7.68\left(\mathrm{~m}, 4 \mathrm{H}, 4 \times \mathrm{CH}_{\text {ortho }}\right.$ ), 7.50-7.44 $\left(\mathrm{m}, 2 \mathrm{H}, 2 \times \mathrm{CH}_{\text {para }}\right), 7.44-7.37\left(\mathrm{~m}, 4 \mathrm{H}, 4 \times \mathrm{CH}_{\text {meta }}\right), 5.84-5.80(\mathrm{~m}, 2 \mathrm{H}$, $\mathrm{H}-2, \mathrm{H}-3$ ), 5.34 (br s, 1H, H-1), 4.90-4.88 (m, 1H, H-4), 3.92 (d, $J=6.2 \mathrm{~Hz}, 1 \mathrm{H}, \mathrm{H}-5), 2.07$ (s, 3H, $\left.\mathrm{CH}_{3}\right), 1.08\left(\mathrm{~s}, 9 \mathrm{H}, 3 \times \mathrm{CH}_{3}\right), 1.07$ (s, 3H, $\left.\mathrm{CH}_{3} \mathrm{COO}\right) .{ }^{13} \mathrm{C}$ NMR (101 MHz, DMSO-d $\left.)_{6}\right) \delta 170.0(\mathrm{C}=\mathrm{O})$, $135.6\left(\mathrm{CH}_{\text {ortho }}\right), 132.1$ (CSi), $130.3(\mathrm{C}-2), 129.9\left(\mathrm{CH}_{\text {para }}\right), 128.9(\mathrm{C}-$ 3), $127.5\left(\mathrm{CH}_{\text {meta }}\right), 78.8$ (C-1), 71.7 (C-5), 69.4 (C-4), 26.8 $\left(\mathrm{C}\left(\mathrm{CH}_{3}\right)_{3}\right), 20.8\left(\mathrm{CH}_{3} \mathrm{COO}\right), 19.1\left(\mathrm{C}\left(\mathrm{CH}_{3}\right)_{3}\right), 18.3\left(\mathrm{CH}_{3}\right)$.

5.3.2.5.2. $\quad N$-[1-(4-O-Acetyl-6-anhydro-2,3-dideoxy- $\beta$-L-erythrohex-2-enopyranosyl)]-N-(O-tert-butyldiphenylsilyl)sulfamide (3e, anomer $\beta, 16 \%) . \quad{ }^{1} \mathrm{H}$ NMR (400 MHz, DMSO-d $\left.d_{6}\right) \delta 9.10$ (s, 1H, NHOSi), $8.97(\mathrm{~d}, J=8.9 \mathrm{~Hz}, 1 \mathrm{H}, \mathrm{NHCH}), 7.74-7.68\left(\mathrm{~m}, 4 \mathrm{H}, 4 \times \mathrm{CH}_{\text {ortho }}\right), 7.50-$ $7.44\left(\mathrm{~m}, 2 \mathrm{H}, 2 \times \mathrm{CH}_{\text {para }}\right), 7.44-7.37$ ( $\left.\mathrm{m}, 4 \mathrm{H}, 4 \times \mathrm{CH}_{\text {meta }}\right), 5.90-5.85$ (m, 2H, H-2, H-3), 5.32 (br s, 1H, H-1), 4.88-4.85 (m, 1H, H-4), $3.94(\mathrm{~d}, J=6.2 \mathrm{~Hz}, 1 \mathrm{H}, \mathrm{H}-5), 2.07\left(\mathrm{~s}, 3 \mathrm{H}, \mathrm{CH}_{3}\right), 1.08\left(\mathrm{~s}, 9 \mathrm{H}, 3 \times \mathrm{CH}_{3}\right)$, $1.07\left(\mathrm{~s}, 3 \mathrm{H}, \mathrm{CH}_{3} \mathrm{COO}\right) .{ }^{13} \mathrm{C}$ NMR (101 MHz, DMSO-d 6 ) $\delta 170.0$ $(\mathrm{C}=\mathrm{O}), 135.6\left(\mathrm{CH}_{\text {ortho }}\right), 132.1(\mathrm{CSi}), 130.3(\mathrm{C}-2), 129.9\left(\mathrm{CH}_{\text {para }}\right)$, 128.9 (C-3), $127.5\left(\mathrm{CH}_{\text {meta }}\right), 76.2(\mathrm{C}-1), 69.6(\mathrm{C}-4), 65.0$ (C-5), 26.8 $\left(\mathrm{C}\left(\mathrm{CH}_{3}\right)_{3}\right), 20.8\left(\mathrm{CH}_{3} \mathrm{COO}\right), 19.1\left(\mathrm{C}\left(\mathrm{CH}_{3}\right)_{3}\right), 18.3\left(\mathrm{CH}_{3}\right)$.

\subsubsection{General procedure for the synthesis of compound 4}

To a solution of compound $3(0.63 \mathrm{mmol})$ in $7 \mathrm{ml}$ of $\mathrm{CH}_{3} \mathrm{CN}$ was added 1.7 equiv of HF.pyr complex (70\%) at room temperature for $3 \mathrm{~h}$. The reaction was then quenched by addition of $\mathrm{Et}_{3} \mathrm{~N}(5 \mathrm{ml})$ and the mixture was concentrated under vacuum. The crude product was then purified by silica gel column chromatography using diethyl ether and pentane as eluent $\left(\mathbf{4 a}, \mathbf{4 b}\right.$ and $\mathbf{4 e}$ : $\mathrm{Et}_{2} \mathrm{O} /$ pentane $7: 3 ; \mathbf{4 c}$ and $\mathbf{4 d ~ E t}{ }_{2} \mathrm{O} /$ pentane $8: 2$ ). The expected compound $\mathbf{4}$ (mixture $\alpha / \beta$ ) are obtained in $85 \%$ yield.

5.3.3.1. Compound 4a. We introduced $0.353 \mathrm{~g}$ of $\mathbf{3 a}$ and we obtained $0.173 \mathrm{~g}$ (yield: $85 \%$ ). HRMS calcd for $\mathrm{C}_{10} \mathrm{H}_{17} \mathrm{~N}_{2} \mathrm{O}_{8} \mathrm{~S}$ $[\mathrm{M}+\mathrm{H}]^{+}:$325.0706, found $325.0703(-0.3 \mathrm{mDa})$.

5.3.3.1.1. $\quad N-[1-(4,6-D i-O-a c e t y l-2,3-d i d e o x y-\alpha$-D-erythro-hex-2eno-pyranosyl)]-N-hydroxysulfamide (4a, anomer $\alpha, 77 \%) .{ }^{1} \mathrm{H}$ NMR (400 MHz, DMSO-d $\left.d_{6}\right) 6.92(\mathrm{~s}, 1 \mathrm{H}, \mathrm{NHOH}), 6.69(\mathrm{~s}, 1 \mathrm{H}$, $\mathrm{NHCH}$ ), 5.87 (td, $J=10.5,1.8 \mathrm{~Hz}, 1 \mathrm{H}, \mathrm{H}-3$ ), 5.79 (ddd, $J=10.5,3.2$, $2.5 \mathrm{~Hz}, 1 \mathrm{H}, \mathrm{H}-2), 5.24$ (ddd, $J=9.2,2.9,1.8 \mathrm{~Hz}, 1 \mathrm{H}, \mathrm{H}-1$ ), $5.12-$ $5.10(\mathrm{~m}, 1 \mathrm{H}, \mathrm{H}-4), 4.12(\mathrm{dd}, J=12.0,4.1 \mathrm{~Hz}, 1 \mathrm{H}, \mathrm{H}-6 \mathrm{a}), 4.12-4.08$ (m, 1H, NHOH), 4.08-4.05 (m, 1H, H-5), 4.03 (dd, $J=11.6,4.3 \mathrm{~Hz}$, $1 \mathrm{H}, \mathrm{H}-6 \mathrm{~b}), 2.05$ (s, 3H, $\left.\mathrm{CH}_{3} \mathrm{COO}\right), 2.02$ (s, 3H, $\left.\mathrm{CH}_{3} \mathrm{COO}\right) .{ }^{13} \mathrm{C}$ NMR $\left(101 \mathrm{MHz}, \mathrm{DMSO}-d_{6}\right) \delta 170.0(\mathrm{C}=\mathrm{O}), 169.8(\mathrm{C}=\mathrm{O}), 130.4(\mathrm{~s}, 1 \mathrm{H}, \mathrm{C}-$ 3), 127.0 (C-2), 87.5 (C-1), 65.8 (C-5), 64.8 (C-4), 62.8 (C-6), 20.4 $\left(\mathrm{CH}_{3} \mathrm{COO}\right)$.

5.3.3.1.2. $N$-[1-(4,6-Di-O-acetyl-2,3-dideoxy- $\beta$-D-threo-hex-2-enopyranosyl)]-N-hydroxysulfamide (4a, anomer $\beta, 23 \%) .{ }^{1} \mathrm{H}$ NMR $\left(400 \mathrm{MHz}, \mathrm{DMSO}-d_{6}\right): \delta 6.90(\mathrm{~s}, 1 \mathrm{H}, \mathrm{NHOH}), 6.68(\mathrm{~s}, 1 \mathrm{H}, \mathrm{NHCH})$, $5.84(\mathrm{td}, J=10.5,1.6 \mathrm{~Hz}, 1 \mathrm{H}, \mathrm{H}-3), 5.79(\mathrm{td}, J=10.5,1.3 \mathrm{~Hz}, 1 \mathrm{H}$, $\mathrm{H}-2$ ), 5.33-5.28 (m, 1H, H-1), 5.14-5.12 (m, 1H, H-4), 4.12-4.08 (m, 3H, NHOH, H-6a, H-6b), 3.89 (ddd, $J=8.2,4.7,3.2 \mathrm{~Hz}, 1 \mathrm{H}$, $\mathrm{H}-5), 2.04\left(\mathrm{~s}, 3 \mathrm{H}, \mathrm{CH}_{3} \mathrm{COO}\right), 2.03\left(\mathrm{~s}, 3 \mathrm{H}, \mathrm{CH}_{3} \mathrm{COO}\right) .{ }^{13} \mathrm{C} \mathrm{NMR}$ $\left(101 \mathrm{MHz}, \mathrm{DMSO}-d_{6}\right) \delta 170.0(\mathrm{C}=0), 169.8(\mathrm{C}=0), 132.9(\mathrm{C}-3)$, 126.0 (C-2), 90.3 (C-1), 72.1 (C-5), 64.3 (C-4), 62.9 (C-6), 20.6 $\left(\mathrm{CH}_{3} \mathrm{COO}\right)$.

5.3.3.2. Compound $4 \mathbf{b}$. We introduced $0.357 \mathrm{~g}$ of $\mathbf{3 b}$ and we obtained $0.175 \mathrm{~g}$ (yield: 85\%). HRMS calcd for $\mathrm{C}_{10} \mathrm{H}_{17} \mathrm{~N}_{2} \mathrm{O}_{8} \mathrm{~S}$ $[\mathrm{M}+\mathrm{H}]^{+}:$325.0706, found $325.0703(-0.3 \mathrm{mDa})$.

5.3.3.2.1. N-[1-(4,6-Di-O-acetyl-2,3-dideoxy- $\alpha$-D-threo-hex-2-enopyranosyl)]-N-hydroxysulfamide (4b, anomer $\alpha, 77 \%$ ). ${ }^{1} \mathrm{H}$ NMR $\left(400 \mathrm{MHz}, \mathrm{DMSO}-d_{6}\right) \delta 6.92(\mathrm{~s}, 1 \mathrm{H}, \mathrm{NHOH}), 6.69(\mathrm{~s}, 1 \mathrm{H}, \mathrm{NHCH})$, 6.07 (ddd, $J=9.9,5.3,1.4 \mathrm{~Hz}, 1 \mathrm{H}, \mathrm{H}-3$ ), 6.02 (dd, $J=9.9,3.3 \mathrm{~Hz}$, $1 \mathrm{H}, \mathrm{H}-2$ ), 5.42-5.38 (m, 1H, H-1), 4.96 (dd, $J=5.2,2.5 \mathrm{~Hz}, 1 \mathrm{H}, \mathrm{H}-$ 4), 4.40 (dd, $J=6.5,2.5 \mathrm{~Hz}, 1 \mathrm{H}, \mathrm{H}-5), 4.13-4.10(\mathrm{~m}, 1 \mathrm{H}, \mathrm{NHOH})$, 4.09 (dd, $J=12.0,4.1 \mathrm{~Hz}, 1 \mathrm{H}, \mathrm{H}-6 \mathrm{a}), 4.06$ (dd, $J=11.2,6.5 \mathrm{~Hz}, 1 \mathrm{H}$, $\mathrm{H}-6 \mathrm{~b}), 1.95$ (s, 6H, $\left.2 \times \mathrm{CH}_{3} \mathrm{COO}\right) .{ }^{13} \mathrm{C}$ NMR (101 MHz, DMSO-d $\left.d_{6}\right) \delta$ 
$169.8(C=0), 130.6(C-2), 127.3(C-3), 87.5(C-1), 65.9(C-5), 63.0$ (C-4), 62.7 (C-6), $20.4\left(2 \times \mathrm{CH}_{3} \mathrm{COO}\right)$.

5.3.3.2.2. $\quad N$-[1-(4,6-Di-O-acetyl-2,3-dideoxy- $\beta$-D-erythro-hex-2eno-pyranosyl)]-N-hydroxysulfamide (4b, anomer $\beta, 23 \%) . \quad{ }^{1} \mathrm{H}$ NMR $\left(400 \mathrm{MHz}, \mathrm{DMSO}_{6}\right) \delta 6.90(\mathrm{~s}, 1 \mathrm{H}, \mathrm{NHOH}), 6.68(\mathrm{~s}, 1 \mathrm{H}$, $\mathrm{NHCH}$ ), 6.05 (ddd, $J=10.1,5.1,1.6 \mathrm{~Hz}, 1 \mathrm{H}, \mathrm{H}-3$ ), 6.00 (br d, $J=10.5 \mathrm{~Hz}, 1 \mathrm{H}, \mathrm{H}-2), 5.34$ (dd, $J=9.0,4.7 \mathrm{~Hz}, 1 \mathrm{H}, \mathrm{H}-1), 4.96(\mathrm{dd}$, $J=5.0,1.8 \mathrm{~Hz}, 1 \mathrm{H}, \mathrm{H}-4), 4.13-4.10(\mathrm{~m}, 3 \mathrm{H}, \mathrm{NHOH}, \mathrm{H}-6 \mathrm{a}, \mathrm{H}-6 \mathrm{~b})$, 4.08 (dt, $J=6.2,2.4 \mathrm{~Hz}, 1 \mathrm{H}, \mathrm{H}-5), 2.00\left(\mathrm{~s}, 6 \mathrm{H}, 2 \times \mathrm{CH}_{3} \mathrm{COO}\right) .{ }^{13} \mathrm{C}$ NMR (101 MHz, DMSO- $\left.d_{6}\right) \delta 170.0(\mathrm{C}=\mathrm{O}), 133.1(\mathrm{C}-2), 126.1(\mathrm{C}-$ 3), 90.3 (C-1), 72.27 (C-5), 64.9 (C-4), 64.4 (C-6), 20.6 (2× $\left.\mathrm{CH}_{3} \mathrm{COO}\right)$.

5.3.3.3. Compound 4c. We introduced $0.309 \mathrm{~g}$ of $3 \mathrm{c}$ and we obtained $0.135 \mathrm{~g}$ (yield: 85\%). HRMS calcd for $\mathrm{C}_{7} \mathrm{H}_{13} \mathrm{~N}_{2} \mathrm{O}_{6} \mathrm{~S}$ $[\mathrm{M}+\mathrm{H}]^{+}:$253.0494, found $253.0492(-0.2 \mathrm{mDa})$.

5.3.3.3.1. $\quad N-[1-(4-0-A c e t y l-5-a n h y d r o-2,3-d i d e o x y-\alpha$-D-erythropent-2-enopyranosyl)]-N-hydroxysulfamide (4c, anomer $\alpha, 77 \%) .{ }^{1} \mathrm{H}$ NMR (400 MHz, DMSO-d $\left.d_{6}\right) \delta 6.92(\mathrm{~s}, 1 \mathrm{H}, \mathrm{NHOH}), 6.69(\mathrm{~s}, 1 \mathrm{H}, \mathrm{NHCH})$, 6.07-6.02 (m, 1H, H-3), 5.99-5.96 ( $\mathrm{m}, 1 \mathrm{H}, \mathrm{H}-2), 5.36-5.33(\mathrm{~m}, 1 \mathrm{H}, \mathrm{H}-$ 1), $4.90-4.84(\mathrm{~m}, 1 \mathrm{H}, \mathrm{H}-4), 4.22-4.13(\mathrm{~m}, 2 \mathrm{H}, \mathrm{H}-5 \mathrm{a}, \mathrm{H}-5 \mathrm{~b}), 3.85-3.69$ $(\mathrm{m}, 1 \mathrm{H}, \mathrm{NHOH}), 2.03\left(\mathrm{~s}, 3 \mathrm{H}, \mathrm{CH}_{3} \mathrm{COO}\right) .{ }^{13} \mathrm{C} \mathrm{NMR}\left(101 \mathrm{MHz}, \mathrm{DMSO}-d_{6}\right)$ $\delta 170.2$ (C=0), 130.9 (C-2), 125.1 (C-3), 87.6 (C-1), 63.2 (C-4), 61.8 (C-5), $21.04\left(\mathrm{CH}_{3} \mathrm{COO}\right)$.

5.3.3.3.2. $\mathrm{N}$-[1-(4-O-Acetyl-5-anhydro-2,3-dideoxy- $\beta$-D-threo-pent2-enopyranosyl)]-N-hydroxysulfamide (4c, anomer $\beta, 23 \%) . \quad{ }^{1} \mathrm{H}$ NMR $\left(400 \mathrm{MHz}, \mathrm{DMSO}-d_{6}\right) \delta 6.90(\mathrm{~s}, 1 \mathrm{H}, \mathrm{NHOH}), 6.68(\mathrm{~s}, 1 \mathrm{H}, \mathrm{NHCH})$, 6.07-6.02 (m, 1H, H-3), 5.88 (ddd, $J=10.1,2.2,1.3 \mathrm{~Hz}, 1 \mathrm{H}, \mathrm{H}-2$ ), $5.26(\mathrm{dd}, J=9.0,2.0 \mathrm{~Hz}, 1 \mathrm{H}, \mathrm{H}-1), 4.90-4.84(\mathrm{~m}, 1 \mathrm{H}, \mathrm{H}-4), 3.85-$ 3.69 ( $\mathrm{m}, 3 \mathrm{H}, \mathrm{H}-5 \mathrm{a}, \mathrm{H}-5 \mathrm{~b}, \mathrm{NHOH}), 2.03$ (s, 3H, $\left.\mathrm{CH}_{3} \mathrm{COO}\right) .{ }^{13} \mathrm{C}$ NMR (101 MHz, DMSO-d $\left.d_{6}\right) \delta 170.3(\mathrm{C}=0), 130.6(\mathrm{C}-2), 127.8$ (C-3), 90.5 (C-1), 64.1 (C-4), 62.2 (C-5), $21.0\left(\mathrm{CH}_{3} \mathrm{COO}\right)$.

5.3.3.4. Compound 4d. We introduced $0.311 \mathrm{~g}$ of $3 \mathrm{~d}$ and we obtained $0.136 \mathrm{~g}$ (yield: 85\%). HRMS calcd for $\mathrm{C}_{7} \mathrm{H}_{13} \mathrm{~N}_{2} \mathrm{O}_{6} \mathrm{~S}$ $[\mathrm{M}+\mathrm{H}]^{+}:$253.0494, found $253.0494(0.0 \mathrm{mDa})$.

5.3.3.4.1. $\mathrm{N}$-[1-(4-O-Acetyl-5-anhydro-2,3-dideoxy- $\alpha$-L-threo-pent2-enopyranosyl)]-N-hydroxysulfamide (4d, anomer $\alpha, 77 \%) . \quad{ }^{1} \mathrm{H}$ NMR $\left(400 \mathrm{MHz}, \mathrm{DMSO}-d_{6}\right) \delta 6.92(\mathrm{~s}, 1 \mathrm{H}, \mathrm{NHOH}), 6.69(\mathrm{~s}, 1 \mathrm{H}, \mathrm{NHCH}), 6.08-$ $6.01(\mathrm{~m}, 1 \mathrm{H}, \mathrm{H}-3), 6.00-5.97(\mathrm{~m}, 1 \mathrm{H}, \mathrm{H}-2), 5.35$ (dd, $J=9.0,1.8 \mathrm{~Hz}$, $1 \mathrm{H}, \mathrm{H}-1), 4.89-4.84$ (m, 1H, H-4), 4.22-4.13 (m, 2H, H-5a, H-5b), 3.84-3.70 (m, 1H, NHOH), $2.04\left(\mathrm{~s}, 3 \mathrm{H}, \mathrm{CH}_{3} \mathrm{COO}\right) .{ }^{13} \mathrm{C}$ NMR (101 MHz, DMSO-d $\left.d_{6}\right) \delta 170.2(\mathrm{C}=0), 131.0(\mathrm{C}-2), 125.2$ (C-3), 87.4 (C-1), 63.3 (C-4), 61.9 (C-5), $21.05\left(\mathrm{CH}_{3} \mathrm{COO}\right)$.

5.3.3.4.2. $\quad N$-[1-(4-O-Acetyl-5-anhydro-2,3-dideoxy- $\beta$-L-erythropent-2-enopyranosyl)]-N-hydroxysulfamide (4d, anomer $\beta, 23 \%) . \quad{ }^{1} \mathrm{H}$ NMR (400 MHz, DMSO-d $\left.d_{6}\right) \delta 6.90(\mathrm{~s}, 1 \mathrm{H}, \mathrm{NHOH}), 6.68(\mathrm{~s}, 1 \mathrm{H}, \mathrm{NHCH})$, 6.08-6.01 (m, 1H, H-3), 6.00-5.97 (m, 1H, H-2), 5.27 (dd, J=9.0, $2.0 \mathrm{~Hz}, 1 \mathrm{H}, \mathrm{H}-1), 4.88-4.85(\mathrm{~m}, 1 \mathrm{H}, \mathrm{H}-4), 3.84-3.70(\mathrm{~m}, 2 \mathrm{H}, \mathrm{H}-5 \mathrm{a}$, $\mathrm{H}-5 \mathrm{~b}, \mathrm{NHOH}), 2.03\left(\mathrm{~s}, 3 \mathrm{H}, \mathrm{CH}_{3} \mathrm{COO}\right) .{ }^{13} \mathrm{C}$ NMR (101 MHz, DMSO- $\left.d_{6}\right)$ $\delta 170.3(\mathrm{C}=\mathrm{O}), 130.3(\mathrm{C}-2), 127.8(\mathrm{C}-3), 90.3(\mathrm{C}-1), 64.2(\mathrm{C}-4), 62.2$ (C-5), $21.00\left(\mathrm{CH}_{3} \mathrm{COO}\right)$.

5.3.3.5. Compound 4e. We introduced $0.318 \mathrm{~g}$ of $\mathbf{3 e}$ and we obtained $0.143 \mathrm{~g}$ (yield: 85\%). HRMS calcd for $\mathrm{C}_{8} \mathrm{H}_{15} \mathrm{~N}_{2} \mathrm{O}_{6} \mathrm{~S}$ $[\mathrm{M}+\mathrm{H}]^{+}:$267.0651, found $267.0654(0.3 \mathrm{mDa})$.

5.3.3.5.1. $\quad N$-[1-(4-O-Acetyl-6-anhydro-2,3-dideoxy- $\alpha$-L-erythrohex-2-enopyranosyl)]-N-hydroxysulfamide (4e, anomer $\alpha, 77 \%$ ). ${ }^{1} \mathrm{H}$ NMR $\left(400 \mathrm{MHz}\right.$, DMSO- $\left.d_{6}\right) \delta 6.92(\mathrm{~s}, 1 \mathrm{H}, \mathrm{NHOH}), 6.69(\mathrm{~s}, 1 \mathrm{H}$, $\mathrm{NHCH}), 5.90-5.85$ (m, 2H, H-2, H-3), 5.32 (br s, 1H, H-1), 4.88$4.85(\mathrm{~m}, 1 \mathrm{H}, \mathrm{H}-4), 3.98-3.89(\mathrm{~m}, 2 \mathrm{H}, \mathrm{H}-5, \mathrm{NHOH}), 2.07$ (s, 3H, $\left.\mathrm{CH}_{3}\right), 1.07\left(\mathrm{~s}, 3 \mathrm{H}, \mathrm{CH}_{3} \mathrm{COO}\right) .{ }^{13} \mathrm{C}$ NMR $\left(101 \mathrm{MHz}, \mathrm{DMSO}-\mathrm{d}_{6}\right) \delta$ 170.0 ( $\mathrm{C}=0$ ), 130.2 (C-2), 127.5 (C-3), 87.4 (C-1), 69.6 (C-4), 65.1 (C-5), $20.8\left(\mathrm{CH}_{3} \mathrm{COO}\right), 19.1\left(\mathrm{CH}_{3}\right)$.

5.3.3.5.2. $N$-[1-(4-O-Acetyl-6-anhydro-2,3-dideoxy- $\beta$-L-threo-hex2-enopyranosyl)]-N-hydroxysulfamide (4e, anomer $\beta, 23 \%) .{ }^{1} \mathrm{H}$ NMR (400 MHz, DMSO-d $\left.d_{6}\right) \delta 6.90(\mathrm{~s}, 1 \mathrm{H}, \mathrm{NHOH}), 6.68(\mathrm{~s}, 1 \mathrm{H}$,
NHCH), 5.84-5.80 (m, 2H, H-2, H-3), 5.34 (br s, 1H, H-1), 4.90$4.88(\mathrm{~m}, 1 \mathrm{H}, \mathrm{H}-4), 3.98-3.89(\mathrm{~m}, 2 \mathrm{H}, \mathrm{H}-5, \mathrm{NHOH}), 2.07(\mathrm{~s}, 3 \mathrm{H}$, $\left.\mathrm{CH}_{3}\right), 1.07\left(\mathrm{~s}, 3 \mathrm{H}, \mathrm{CH}_{3} \mathrm{COO}\right) .{ }^{13} \mathrm{C}$ NMR $\left(101 \mathrm{MHz}\right.$, DMSO- $\left.d_{6}\right) \delta$ 170.0 (C=0), 130.2 (C-2), 127.5 (C-3), 90.3 (C-1), 69.6 (C-4), 65.1 (C-5), $20.8\left(\mathrm{CH}_{3} \mathrm{COO}\right), 19.1\left(\mathrm{CH}_{3}\right)$.

\subsubsection{General procedure for the synthesis of compound 5}

Compound $4(0.318 \mathrm{mmol})$ were dissolved in a $2.0 \mathrm{M} \mathrm{NH}_{3}$ methanolic solution $(8 \mathrm{ml})$ at $0-5{ }^{\circ} \mathrm{C}$. The reaction was stirred at $4{ }^{\circ} \mathrm{C}$ overnight, then concentrated under vacuum to give compound 5 ( $\alpha$ and $\beta$ anomers mixture) in quantitative yield.

5.3.4.1. Compound 5a. We introduced $0.103 \mathrm{~g}$ of $\mathbf{4 a}$ and we obtained $0.076 \mathrm{~g}$ (yield: $100 \%$ ). HRMS calcd for $\mathrm{C}_{6} \mathrm{H}_{13} \mathrm{~N}_{2} \mathrm{O}_{6} \mathrm{~S}$ $[\mathrm{M}+\mathrm{H}]^{+}:$241.0494, found $241.0496(0.2 \mathrm{mDa})$.

5.3.4.1.1. $N$-[1-(2,3-Dideoxy- $\alpha$-D-erythro-hex-2-enopyranosyl)]-Nhydroxysulfamide (5a, anomer $\alpha, 77 \%) . \quad{ }^{1} \mathrm{H}$ NMR $\left(400 \mathrm{MHz}, \mathrm{D}_{2} \mathrm{O}\right) \delta$ $6.92(\mathrm{~s}, 1 \mathrm{H}, \mathrm{NHOH}), 6.69(\mathrm{~s}, 1 \mathrm{H}, \mathrm{NHCH}), 5.90-5.88(\mathrm{~m}, 1 \mathrm{H}, \mathrm{H}-3)$, 5.85-5.83 (m, 1H, H-2), 5.29 (ddd, $J=9.4,3.5,1.7 \mathrm{~Hz}, 1 \mathrm{H}, \mathrm{H}-1$ ), 4.22 (dd, $J=8.8,2.2 \mathrm{~Hz}, 1 \mathrm{H}, \mathrm{H}-4$ ), 3.89-3.87 ( $\mathrm{m}, 4 \mathrm{H}, \mathrm{H}-5, \mathrm{H}-6 \mathrm{a}, \mathrm{H}-$ 6b, NHOH), 3.70-3.65 (m, 2H, OH). ${ }^{13} \mathrm{C}$ NMR (101 MHz, $\left.\mathrm{D}_{2} \mathrm{O}\right) \delta$ 130.4 (C-3), 127.0 (C-2), 87.5 (C-1), 78.1 (C-5), 68.3 (C-4), 60.0 (C-6).

5.3.4.1.2. $N$-[1-(2,3-Dideoxy- $\beta$-D-threo-hex-2-eno-pyranosyl)]-Nhydroxysulfamide (5a, anomer $\beta, 23 \%) . \quad{ }^{1} \mathrm{H}$ NMR $\left(400 \mathrm{MHz}, \mathrm{D}_{2} \mathrm{O}\right)$ $\delta 6.90(\mathrm{~s}, 1 \mathrm{H}, \mathrm{NHOH}), 6.68(\mathrm{~s}, 1 \mathrm{H}, \mathrm{NHCH}), 5.90-5.88(\mathrm{~m}, 1 \mathrm{H}, \mathrm{H}-3)$, 5.85-5.83 (m, $1 \mathrm{H}, \mathrm{H}-2), 5.32-5.26(\mathrm{~m}, 1 \mathrm{H}, \mathrm{H}-1), 4.24$ (dd, $J=7.7$, $1.5 \mathrm{~Hz}, 1 \mathrm{H}, \mathrm{H}-4), 3.89-3.87(\mathrm{~m}, 4 \mathrm{H}, \mathrm{H}-5, \mathrm{H}-6 \mathrm{a}, \mathrm{H}-6 \mathrm{~b}, \mathrm{NHOH})$ 3.70-3.65 (m, 2H, OH). ${ }^{13} \mathrm{C}$ NMR (101 MHz, $\left.\mathrm{D}_{2} \mathrm{O}\right) \delta 130.35$ (C-3), 127.02 (C-2), 90.34 (C-1), 78.11 (C-5), 68.78 (C-4), 60.03 (C-6).

5.3.4.2. Compound 5b. We introduced $0.103 \mathrm{~g}$ of $\mathbf{4 b}$ and we obtained $0.077 \mathrm{~g}$ (yield: 100\%). HRMS calcd for $\mathrm{C}_{6} \mathrm{H}_{13} \mathrm{~N}_{2} \mathrm{O}_{6} \mathrm{~S}$ $[\mathrm{M}+\mathrm{H}]^{+}:$241.0494, found $241.0496(0.2 \mathrm{mDa})$.

5.3.4.2.1. $\quad N-[1-(2,3-D i d e o x y-\alpha-D-t h r e o-h e x-2-e n o-p y r a n o s y l)]-N-$ hydroxysulfamide (5b, anomer $\alpha, 77 \%) . \quad{ }^{1} \mathrm{H}$ NMR $\left(400 \mathrm{MHz}, \mathrm{D}_{2} \mathrm{O}\right)$ $\delta 6.92(\mathrm{~s}, 1 \mathrm{H}, \mathrm{NHOH}), 6.69(\mathrm{~s}, 1 \mathrm{H}, \mathrm{NHCH}), 5.90-5.88(\mathrm{~m}, 1 \mathrm{H}, \mathrm{H}-3)$, 5.85-5.83 (m, 1H, H-2), 5.26-5.22 (m, 1H, H-1), 4.22 (dd, J=6.0, $2.1 \mathrm{~Hz}, 1 \mathrm{H}, \mathrm{H}-4)$ 3.93-3.88 (m, 4H, H-5, H-6a, H-6b, NHOH), 3.70-3.65 (m, 2H, OH). ${ }^{13} \mathrm{C}$ NMR $\left(101 \mathrm{MHz}, \mathrm{D}_{2} \mathrm{O}\right) \delta 130.2(\mathrm{C}-3)$, 127.8 (C-2), 88.3 (C-1), 78.0 (C-5), 68.2 (C-4), 60.1 (C-6).

5.3.4.2.2. $\quad N-[1-(2,3-D i d e o x y-\beta$-D-erythro-hex-2-eno-pyranosyl)]$N$-hydroxysulfamide (5b, anomer $\beta, 23 \%) .{ }^{1} \mathrm{H}$ NMR $(400 \mathrm{MHz}$, $\left.\mathrm{D}_{2} \mathrm{O}\right) \delta 6.90(\mathrm{~s}, 1 \mathrm{H}, \mathrm{NHOH}), 6.68(\mathrm{~s}, 1 \mathrm{H}, \mathrm{NHCH}), 5.90-5.88(\mathrm{~m}, 1 \mathrm{H}$, $\mathrm{H}-3), 5.85-5.83(\mathrm{~m}, 1 \mathrm{H}, \mathrm{H}-2), 5.33-5.28(\mathrm{~m}, 1 \mathrm{H}, \mathrm{H}-1), 4.24(\mathrm{dd}$, $J=6.2,1.9 \mathrm{~Hz}, 1 \mathrm{H}, \mathrm{H}-4), 3.93-3.88$ (m, 4H, H-5, H-6a, H-6b, NHOH), 3.70-3.65 (m, 2H, OH). ${ }^{13} \mathrm{C}$ NMR $\left(101 \mathrm{MHz}, \mathrm{D}_{2} \mathrm{O}\right) \delta 130.2(\mathrm{C}-3)$, 127.8 (C-2), 91.0 (C-1), 78.0 (C-5), 68.8 (C-4), 60.1 (C-6).

5.3.4.3. Compound 5c. We introduced $0.080 \mathrm{~g}$ of $\mathbf{4 c}$ and we obtained $0.067 \mathrm{~g}$ (yield: $100 \%$ ). HRMS calcd for $\mathrm{C}_{5} \mathrm{H}_{11} \mathrm{~N}_{2} \mathrm{O}_{5} \mathrm{~S}$ $[\mathrm{M}+\mathrm{H}]^{+}:$211.0389, found $211.0386(0.3 \mathrm{mDa})$.

5.3.4.3.1. $\quad N$-[1-(5-Anhydro-2,3-dideoxy- $\alpha$-D-erythro-pent-2-enopyranosyl)]-N-hydroxysulfamide (5c, anomer $\alpha, 77 \%$ ). ${ }^{1} \mathrm{H}$ NMR $\left(400 \mathrm{MHz}, \mathrm{D}_{2} \mathrm{O}\right) \delta 7.32(\mathrm{~s}, 1 \mathrm{H}, \mathrm{NHOH}), 7.09(\mathrm{~s}, 1 \mathrm{H}, \mathrm{NHCH}), 5.98-$ $5.96(\mathrm{~m}, 1 \mathrm{H}, \mathrm{H}-3), 5.96-5.94(\mathrm{~m}, 1 \mathrm{H}, \mathrm{H}-2), 5.47-5.45(\mathrm{~m}, 1 \mathrm{H}, \mathrm{H}-$ 1), 4.61-4.59 (m, 1H, H-4), 4.18-3.99 (m, 3H, H-5a, H-5b, NHOH), 3.55 (br s, $1 \mathrm{H}, \mathrm{NHOH}) .{ }^{13} \mathrm{C}$ NMR $\left(101 \mathrm{MHz}, \mathrm{D}_{2} \mathrm{O}\right) \delta 130.8$ (C-2), 127.6 (C-3), 88.0 (C-1), 64.0 (C-4), 63.3 (C-5).

5.3.4.3.2. $\mathrm{N}$-[1-(5-Anhydro-2,3-dideoxy- $\beta$-D-threo-pent-2-enopyranosyl)]-N-hydroxysulfamide (5c, anomer $\beta, \quad 23 \%) . \quad{ }^{1} \mathrm{H} \quad \mathrm{NMR}$ $\left(400 \mathrm{MHz}, \mathrm{D}_{2} \mathrm{O}\right) \delta 7.30(\mathrm{~s}, 1 \mathrm{H}, \mathrm{NHOH}), 7.08(\mathrm{~s}, 1 \mathrm{H}, \mathrm{NHCH}), 5.98-$ $5.96(\mathrm{~m}, 1 \mathrm{H}, \mathrm{H}-3), 5.96-5.94(\mathrm{~m}, 1 \mathrm{H}, \mathrm{H}-2), 5.44-5.43(\mathrm{~m}, 1 \mathrm{H}, \mathrm{H}-$ 1), 4.65-4.64 (m, 1H, H-4), 4.18-3.99 (m, 3H, H-5a, H-5b, NHOH), 3.55 (br s, $1 \mathrm{H}, \mathrm{NHOH}) .{ }^{13} \mathrm{C}$ NMR $\left(101 \mathrm{MHz}, \mathrm{D}_{2} \mathrm{O}\right) \delta 130.8$ (C-2), 127.6 (C-3), 92.0 (C-1), 64.0 (C-4), 63.3 (C-5). 
5.3.4.4. Compound 5d. We introduced $0.080 \mathrm{~g}$ of $\mathbf{4 d}$ and we obtained $0.067 \mathrm{~g}$ (yield: $100 \%$ ). HRMS calcd for $\mathrm{C}_{5} \mathrm{H}_{12} \mathrm{~N}_{2} \mathrm{O}_{5} \mathrm{~S}$ $[\mathrm{M}+\mathrm{H}]^{+}:$211.0389, found $211.0388(-0.1 \mathrm{mDa})$.

5.3.4.4.1. $\quad N$-[1-(5-Anhydro-2,3-dideoxy- $\alpha$-L-threo-pent-2-enopyranosyl)]-N-hydroxysulfamide (5d, anomer $\alpha, 77 \%) .{ }^{1} \mathrm{H}$ NMR $\left(400 \mathrm{MHz}, \mathrm{D}_{2} \mathrm{O}\right) \delta 7.29(\mathrm{~s}, 1 \mathrm{H}, \mathrm{NHOH}), 6.99(\mathrm{~s}, 1 \mathrm{H}, \mathrm{NHCH}), 5.95-$ $5.90(\mathrm{~m}, 1 \mathrm{H}, \mathrm{H}-3), 5.89-5.85(\mathrm{~m}, 1 \mathrm{H}, \mathrm{H}-2), 5.38-5.35(\mathrm{~m}, 1 \mathrm{H}, \mathrm{H}-$ 1), 4.69-4.63 (m, 1H, H-4), 4.21-4.13 (m, 3H, H-5a, H-5b, NHOH), 3.86 (br s, $1 \mathrm{H}, \mathrm{NHOH}) .{ }^{13} \mathrm{C}$ NMR (101 MHz, $\left.\mathrm{D}_{2} \mathrm{O}\right) \delta 130.52$ (C-2), 127.64 (C-3), 87.16 (C-1), 66.09 (C-4), 63.42 (C-5).

5.3.4.4.2. $\quad N$-[1-(5-Anhydro-2,3-dideoxy- $\beta$-L-erythro-pent-2-enopyranosyl)]-N-hydroxysulfamide (5d, anomer $\beta, 23 \%) . \quad{ }^{1} \mathrm{H}$ NMR $\left(400 \mathrm{MHz}, \mathrm{D}_{2} \mathrm{O}\right) \delta 7.26(\mathrm{~s}, 1 \mathrm{H}, \mathrm{NHOH}), 6.96(\mathrm{~s}, 1 \mathrm{H}, \mathrm{NHCH}), 5.95-$ $5.90(\mathrm{~m}, 1 \mathrm{H}, \mathrm{H}-3), 5.89-5.85(\mathrm{~m}, 1 \mathrm{H}, \mathrm{H}-2), 5.38-5.35(\mathrm{~m}, 1 \mathrm{H}, \mathrm{H}-$ 1), 4.63-451 (m, 1H, H-4), 4.21-4.13 (m, 3H, H-5a, H-5b, NHOH), 3.86 (br s, $1 \mathrm{H}, \mathrm{NHOH}) .{ }^{13} \mathrm{C}$ NMR (101 MHz, $\left.\mathrm{D}_{2} \mathrm{O}\right) \delta 130.52$ (C-2), 127.76 (C-3), 91.54 (C-1), 66.09 (C-4), 63.42 (C-5).

5.3.4.5. Compound 5e. We introduced $0.085 \mathrm{~g}$ of $\mathbf{4 e}$ and we obtained $0.072 \mathrm{~g}$ (yield: $100 \%$ ). HRMS calcd for $\mathrm{C}_{6} \mathrm{H}_{13} \mathrm{~N}_{2} \mathrm{O}_{5} \mathrm{~S}$ $[\mathrm{M}+\mathrm{H}]^{+}:$225.0545, found $225.0543(-0.2 \mathrm{mDa})$.

5.3.4.5.1. $N$-[1-(6-Anhydro-2,3-dideoxy- $\alpha$-L-threo-hex-2-eno-pyranosyl)]-N-hydroxysulfamide (5e, anomer $\alpha, 77 \%) . \quad{ }^{1} \mathrm{H}$ NMR $(400 \mathrm{MHz}$, $\left.\mathrm{D}_{2} \mathrm{O}\right) \delta 6.92(\mathrm{~s}, 1 \mathrm{H}, \mathrm{NHOH}), 6.69(\mathrm{~s}, 1 \mathrm{H}, \mathrm{NHCH}), 6.07(\mathrm{~m}, 1 \mathrm{H}$, $\mathrm{H}-3), 6.02-5.98$ ( $\mathrm{m}, 1 \mathrm{H}, \mathrm{H}-2), 5.41-5.39$ ( $\mathrm{m}, 1 \mathrm{H}, \mathrm{H}-1), 4.45-4.40$ (m, $1 \mathrm{H}, \mathrm{H}-4), 3.98-3.89(\mathrm{~m}, 2 \mathrm{H}, \mathrm{H}-5, \mathrm{NHOH}), 2.00$ (br s, $\left.3 \mathrm{H}, \mathrm{CH}_{3}\right) .{ }^{13} \mathrm{C}$ NMR (101 MHz, D $\left.\mathrm{D}_{2} \mathrm{O}\right) \delta 130.1$ (C-2), 126.9 (C-3), 87.4 (C-1), 70.5 (C-4), $66.0(\mathrm{C}-5), 19.6\left(\mathrm{CH}_{3}\right)$.

5.3.4.5.2. N-[1-(6-Anhydro-2,3-dideoxy- $\beta$-L-erythro-hex-2-enopyranosyl)]-N-hydroxysulfamide (5e, anomer $\beta, 23 \%$ ). We introduced $0.085 \mathrm{~g}$ of $4 \mathbf{e}$ and we obtained $0.072 \mathrm{~g}$ (yield: $100 \%$ ). ${ }^{1} \mathrm{H}$ $\operatorname{NMR}\left(400 \mathrm{MHz}, \mathrm{D}_{2} \mathrm{O}\right) \delta 6.90(\mathrm{~s}, 1 \mathrm{H}, \mathrm{NHOH}), 6.68(\mathrm{~s}, 1 \mathrm{H}, \mathrm{NHCH})$, $6.07(\mathrm{~m}, 1 \mathrm{H}, \mathrm{H}-3), 6.02-5.98(\mathrm{~m}, 1 \mathrm{H}, \mathrm{H}-2), 5.39-5.37(\mathrm{~m}, 1 \mathrm{H}, \mathrm{H}-$ 1), 4.39-4.37 (m, 1H, H-4), 3.97-3.86 (m, 2H, H-5, NHOH, OH), 2.00 (br s, 3H, $\left.\mathrm{CH}_{3}\right) .{ }^{13} \mathrm{C}$ NMR (101 MHz, $\left.\mathrm{D}_{2} \mathrm{O}\right) \delta 130.1$ (C-2), 124.9 (C-3), 90.4 (C-1), 70.5 (C-4), 66.0 (C-5), $19.6\left(\mathrm{CH}_{3}\right)$.

\subsection{CA inhibition assays}

$\mathrm{CO}_{2}$ hydrase assay: an Applied Photophysics stopped-flow instrument has been used for assaying the CA-catalyzed $\mathrm{CO}_{2}$ hydration activity. ${ }^{13}$ Phenol red (at a concentration of $0.2 \mathrm{mM}$ ) has been used as an indicator, working at the absorbance maximum of $557 \mathrm{~nm}$, with $20 \mathrm{mM}$ Hepes (pH 7.5) as buffer, and $20 \mathrm{mM} \mathrm{Na}_{2} \mathrm{SO}_{4}$ (for maintaining constant the ionic strength), following the initial rates of the $\mathrm{CA}$-catalyzed $\mathrm{CO}_{2}$ hydration reaction for a period of $10-100 \mathrm{~s}$. The $\mathrm{CO}_{2}$ concentrations ranged from 1.7 to $17 \mathrm{mM}$ for the determination of the kinetic parameters and inhibition constants. For each inhibitor, at least six traces of the initial 5$10 \%$ of the reaction have been used for determining the initial velocity. The uncatalyzed rates were determined in the same manner and subtracted from the total observed rates. Stock solutions of inhibitor $(0.1 \mathrm{mM})$ were prepared in distilled-deionized water, and dilutions up to $0.01 \mathrm{nM}$ were done thereafter with distilled-deionized water. Inhibitor and enzyme solutions were preincubated together for $15 \mathrm{~min}$ at room temperature prior to assay, to allow for the formation of the E-I complex. The inhibition constants were obtained by nonlinear least squares methods using the Cheng-Prusoff equation and represent the mean from at least three different determinations. Errors were in the range of $\pm 5-10 \%$ of the reported $K_{\mathrm{I}}$ values. CA isoforms were recombinant enzymes obtained in house as reported earlier. ${ }^{14-16}$ The enzyme concentrations in the assay system were: hCA I: $13.2 \mathrm{nM}$; hCA II: $8.4 \mathrm{nM}$; hCA IX: 7.9 nM; hCA XII: 15.2 nM; hCA XIV: 10.7 nM.

\section{Acknowledgments}

The authors would like to thank the Gabonese Ministry of Research for Ph-D fellowship (to J.O.) and the CNRS/CNR (CoopIntEER Program, Grant No. 131999, to J.-Y.W.) for financial support.

\section{Supplementary data}

Supplementary data $\left({ }^{1} \mathrm{H}\right.$ NMR and ${ }^{13} \mathrm{C}$ NMR spectra of products) associated with this article can be found, in the online version, at http://dx.doi.org/10.1016/j.bmc.2014.09.053.

\section{References and notes}

1. (a)Winum, J.-Y.; Colinas, P. A.; Supuran, C. T. Bioorg. Med. Chem. 2013, 21, 1419; (b) Winum, J.-Y.; Poulsen, S. A.; Supuran, C. T. Med. Res. Rev. 2009, 29, 419.

2. (a) Touisni, N.; Maresca, A.; McDonald, P. C.; Lou, Y.; Scozzafava, A.; Dedhar, S.; Winum, J.-Y.; Supuran, C. T. J. Med. Chem. 2011, 54, 8271; (b) Lou, Y.; McDonald, P. C.; Oloumi, V.; Chia, S.; Ostlund, C.; Ahmadi, A.; Kyle, A.; Auf dem Keller, U.; Leung, S.; Huntsman, D.; Clarke, B.; Sutherland, B. W.; Waterhouse, D.; Bally, M.; Roskelley, C.; Overall, C. M.; Minchinton, A.; Pacchiano, F.; Carta, F.; Scozzafava, A.; Touisni, N.; Winum, J.-Y.; Supuran, C. T.; Dedhar, S. Cancer Res. 2011, 71, 3364

3. (a) Lock, F. E.; McDonald, P. C.; Lou, Y.; Serrano, I.; Chafe, S. C.; Ostlund, C.; Aparicio, S.; Winum, J.-Y.; Supuran, C. T.; Dedhar, S. Oncogene 2013, 32, 5210; (b) Supuran, C. T. Nat. Rev. Drug Disc. 2008, 7, 168; (c) Neri, D.; Supuran, C. T. Nat. Rev. Drug Disc. 2011, 10, 767.

4. (a) Winum, J.-Y.; Innocenti, A.; Nasr, J.; Montero, J.-L.; Scozzafava, A.; Vullo, D.; Supuran, C. T. Bioorg. Med. Chem. Lett. 2005, 15, 2353; (b) Temperini, C.; Winum, J.-Y.; Montero, J.-L.; Scozzafava, A.; Supuran, C. T. Bioorg. Med. Chem. Lett. 2007, 17, 2795; (c) Supuran, C. T. J. Enzyme Inhib. Med. Chem. 2012, 27, 759.

5. Colinas, P. A.; Bravo, R. D. Carbohydr. Res. 2007, 342, 2297.

6. (a) Sau, A.; Santra, A.; Misra, A. K. Synlett 2012, 2341; (b) Santra, A.; Guchhait, G.; Misra, A. K. Synlett 2013, 581.

7. (a) Devanathan, K.; Bell, J. A.; Wilkins, P. C.; Jacobs, H. K.; Gopalan, A. S. Tetrahedron Lett. 2007, 48, 8029; (b) Winum, J.-Y.; Innocenti, A.; Nasr, J.; Montero, J.-L.; Scozzafava, A.; Vullo, D.; Supuran, C. T. Bioorg. Med. Chem. Lett. 2005, 15, 2353.

8. Higashibayashi, S.; Shinko, K.; Ishizu, T.; Hashimoto, K.; Shirahama, H.; Nakata, M. Synlett 2000, 1306

9. Shah, S. T. A.; Singh, S.; Guiry, P. J. J. Org. Chem. 2009, 74, 2179.

10. Kitahara, K.; Shimokawa, J.; Fukuyama, T. Chem. Sci. 2014, 5, 904.

11. Ansari, A. A.; Reddy, Y. S.; Vankar, Y. D. Beilstein J. Org. Chem. 2014, 10, 300.

12. Toshima, K.; Ishizuka, T.; Matsuo, G.; Nakata, M.; Kinoshita, M. J. Chem. Soc., Chem. Commun. 1993, 704.

13. Khalifah, R. G. J. Biol. Chem. 1971, 246, 2561.

14. (a) Vitale, R. M.; Alterio, V.: Innocenti, A.; Winum, J. Y: Monti, S. M.: De Simone, G.; Supuran, C. T. J. Med. Chem. 2009, 52, 5990; (b) De Simone, G.; Alterio, V.; Supuran, C. T. Expert Opin. Drug Discov. 2013, 8, 793; (c) Maresca, A.; Vullo, D.; Scozzafava, A.; Manole, G.; Supuran, C. T. J. Enzyme Inhib. Med. Chem. 2013, 28, 392; (d) Maresca, A.; Scozzafava, A.; Vullo, D.; Supuran, C. T. J. Enzyme Inhib. Med. Chem. 2013, 28, 384

15. (a) D'Ambrosio, K.; Smaine, F. Z.; Carta, F.; De Simone, G.; Winum, J-Y.; Supuran, C. T. J. Med. Chem. 2012, 55, 6776; (b) Bonneau, A.; Maresca, A.; Winum, J. Y.; Supuran, C. T. J. Enzyme Inhib. Med. Chem. 2013, 28, 397; (c) Sharma, A.; Tiwari, M.; Supuran, C. T. J. Enzyme Inhib. Med. Chem. 2014, 29, 292.

16. (a) Pacchiano, F.; Carta, F.; McDonald, P. C.; Lou, Y.; Vullo, D.; Scozzafava, A.; Dedhar, S.; Supuran, C. T. J. Med. Chem. 2011, 54, 1896; (b) Supuran, C. T. J. Enzyme Inhib. Med. Chem. 2013, 28, 229; (c) Ebbesen, P.; Pettersen, E. O.; Gorr, T. A.; Jobst, G.; Williams, K.; Kienninger, J.; Wenger, R. H.; Pastorekova, S.; Dubois, L.; Lambin, P.; Wouters, B. G.; Supuran, C. T.; Poellinger, L.; Ratcliffe, P.; Kanopka, A.; Görlach, A.; Gasmann, M.; Harris, A. L.; Maxwell, P.; Scozzafava, A. J. Enzyme Inhib. Med. Chem. 2009, 24, 1. 